1 Mitochondrial fusion regulates proliferation and differentiation in the type II

2 neuroblast lineage in Drosophila

3

4 Dnyanesh Dubal ${ }^{1}$, Prachiti Moghe ${ }^{1,2}$, Bhavin Uttekar ${ }^{1}$ and Richa Rikhy ${ }^{1 *}$

\title{
Affiliation and contact information
}

1. Biology, Indian Institute of Science Education and Research, Homi Bhabha Road, Pashan, Pune, 411008, India Phone: +91-20-25908065

2. Present address: EMBL Heidelberg, Meyerhofstraße 1, 69117 Heidelberg, Germany

*To whom correspondence is addressed: richa@iiserpune.ac.in

14 Key words: mitochondria, Drosophila, neuroblast, differentiation, Opa1, Marf, Notch, Drp1

Abbreviations: neuroblast (NB); intermediate precursor cells (INPs), ganglion mother cell (GMC), Dynamin related protein (Drp1), Optic atrophy protein 1 (Opa1), Mitochondrial assembly regulatory factor (Marf)

\section{Abstract}

22 Optimal mitochondrial function determined by mitochondrial dynamics, morphology and 23 activity is coupled to stem cell differentiation and organism development. However, the

24 mechanisms of interaction of signaling pathways with mitochondrial morphology and 25 activity are not completely understood. We assessed the role of mitochondrial fusion 26 and fission in differentiation of neural stem cells called neuroblasts (NB) in the 27 Drosophila brain. Depletion of mitochondrial inner membrane fusion protein Opa1 and 
mitochondrial outer membrane protein Marf in the Drosophila type II neuroblast lineage led to mitochondrial fragmentation and loss of activity. Opa1 and Marf depletion did not affect the numbers and polarity of type II neuroblasts but led to a decrease in proliferation and differentiation of cells in the lineage. On the contrary, loss of mitochondrial fission protein Drp1 led to mitochondrial fusion but did not show defects in proliferation and differentiation. Depletion of Drp1 along with Opa1 or Marf also led to mitochondrial fusion and suppressed fragmentation, loss of mitochondrial activity, proliferation and differentiation in the type II NB lineage. We found that Notch signaling depletion via the canonical pathway showed mitochondrial fragmentation and loss of differentiation similar to Opa1 mutants. An increase in Notch signaling required mitochondrial fusion for NB proliferation. Further, Drp1 mutants in combination with Notch depletion showed mitochondrial fusion and drove differentiation in the lineage suggesting that fused mitochondria can influence Notch signaling driven differentiation in the type II NB lineage. Our results implicate a crosstalk between Notch signalling, mitochondrial activity and mitochondrial fusion as an essential step in type II NB

\section{Introduction}

Mitochondria are sparse and fragmented in stem cells and occur as an elaborate network in differentiated cells [1-3]. Stem cells largely depend upon glycolysis as an energy source, whereas differentiated cells produce a large amount of ATP by electron transport chain (ETC) activity [1,4,5]. Fused mitochondrial morphology is associated with high membrane potential, increased ETC activity and high ATP production, while low membrane potential, reduced ETC activity and low ATP production is seen in fragmented mitochondria [1]. Mitochondrial architecture is regulated by a balance of

54 fusion and fission events [6]. Proteins belonging to the family of large GTPases are involved in mitochondrial fusion and fission. Optic atrophy 1 (Opa1, or Opa1-like in Drosophila) and Mitofusin or Mitochondrial assembly regulatory factor (Marf in

57 Drosophila or Mitofusin, Mfn in mammals) facilitate inner and outer mitochondrial 
59 mitochondrial fragmentation [7-10]. A balance of levels and activity of these proteins 60 regulates mitochondrial shape in the cell [6]. Further, Opa1 plays a significant role in regulating cristae organization in addition to inner membrane fusion [11]. Opa1 oligomerization and inner membrane cristae organization is important for ETC activity. The presence of elaborate cristae leads to organization of ETC complexes as super complexes and enhances their activity [12]. Hyperfusion of mitochondria protects them from degradation in autophagy and also loss of ETC activity [13-16].

Recent studies show that alteration of mitochondrial dynamics affects signaling pathways such as the Notch signaling pathway during stem cell differentiation. The Notch receptor is a transmembrane protein activated by ligands such as Delta. The Delta-Notch interaction is followed by cleavage of the Notch intracellular domain (NICD) in the signal receiving cell. NICD enters the nucleus and regulates gene expression along with Suppressor of hairless $(\mathrm{Su}(\mathrm{H}))$ by the canonical pathway thereby providing a signal for proliferation or differentiation [17]. Fragmented mitochondrial morphology maintained by Drp1 in ovarian follicle cells in Drosophila is crucial for activating Notch signaling $[18,19]$. Similarly, loss of Opa1 and Mfn leading to mitochondrial fragmentation in mouse embryonic stem cells causes hyperactivation of Notch and reduces differentiation of ESCs into functional cardiomyocytes due to loss of calcium buffering [20]. On the other hand, activation of Notch signaling by depletion of mitochondrial fusion and increasing reactive oxygen species (ROS) enhances differentiation in mammalian neural stem cells [21]. Thus mitochondrial fragmentation along with elevated calcium and reactive oxygen species increase has been found to be involved in Notch signaling in these contexts. It is of interest to understand whether Notch signaling induces appropriate mitochondrial morphology in differentiation.

The Drosophila neural stem cell or neuroblast (NB) differentiation model has

84 been used effectively to identify regulators of steps of differentiation such as stem cell 85 renewal, asymmetric cell division, polarity formation and lineage development. NBs rely 86 on glycolysis and ETC activity for their energy production during differentiation and 87 tumorigenesis [22-24]. Mitochondrial fusion has recently been found to be essential for 88 tumorigenesis [24]. It remains to be studied whether mitochondrial morphology is also 
regulated to provide appropriate activity for differentiation in NBs. The type I NB lineage in the larval brain consists of $90 \mathrm{NBs}$ marked by the expression of the transcription factor Deadpan (Dpn). These NBs divide asymmetrically to give rise to a ganglion mother cells (GMCs) marked by the expression of Prospero (Pros) in the nucleus $[25,26]$. NBs of the type II lineage also express Dpn and are 8 in number [27]. Like the mammalian neural stem cell differentiation type II NBs undergo multiple steps of differentiation by forming transit amplifying cells called intermediate neural precursor cells (INPs). Newly formed INPs are smaller in size as compared to type II NBs and do not express Dpn. INPs undergo a defined series of transcriptional changes to form mature INPs (mINPs). mINPs express Dpn and proliferate to form GMCs that express Pros. GMCs in both the type I and type II lineages finally differentiate into neurons or glia (Figure 1A). Notch signaling regulates type II NB number and differentiation in the type II NB lineage $[28,29]$.

In this study we have assessed the role of mitochondrial morphology proteins Opa1, Marf and Drp1 in regulating type II NB differentiation. We find that Opa1 and Marf mediated mitochondrial fusion is essential for type II NB differentiation. RNAi mediated knockdown of mitochondrial fusion proteins Opa1 and Marf led to mitochondrial fragmentation, loss of mitochondrial activity and defects in differentiation in the type II NB lineage while NB number and polarity remained unaffected. On the other hand there was no defect in differentiation in NBs depleted of mitochondrial fission protein Drp1. Inhibition of mitochondrial fragmentation in Opa1 and Marf mutants by additional depletion of Drp1 suppressed the differentiation defects suggesting that fused mitochondria are essential for type II NB differentiation. Further, Notch depletion led to fragmented mitochondria and loss of differentiation. Increased Notch activity showed mitochondrial fusion and mitochondrial fusion in the type II NB lineage deficient of Notch led to differentiation. Our results show that mitochondrial fusion interacts with Notch signaling to drive differentiation in the type II NB lineage.

\section{Results}




\section{Depletion of Opa1 and Marf leads to mitochondrial fragmentation and depletion of}

\section{Drp1 leads to mitochondrial fusion in type II NBs}

We depleted Opa1, Marf and Drp1 to investigate the effect of perturbation of mitochondrial morphology on NB numbers and differentiation. We expressed multiple RNAi lines and mutants against opa1, marf and drp1 with neuronal Gal4 drivers in different stages of NB differentiation to analyze their effect on lethality and behavior (Figure S1). inscuteable-Gal4, worniu-Gal4, scabrous-Gal4 and prospero-Gal4 were used to deplete Opa1, Marf and Drp1 using RNAi expression and Drp1 using a dominant negative mutant in all NBs. Opa1, Marf and Drp1 depletion by multiple RNAi lines and mutants showed survival of animals until the pupal stage with inscuteableGal4 and worniu-Gal4 and were lethal or showed behavioral defects as adults. The RNAi lines for Opa1 and Marf that gave a stronger defect with inscuteable-Gal4 and worniu-Gal4 and the dominant negative mutant of Drp1, (Drp1 ${ }^{\mathrm{SD}}$ ) [30] were used to deplete these proteins in the type II NB lineage using pointed-Gal4 for further experiments (Figure 1A, S1). opa1 RNAi, marf RNAi and Drp1 ${ }^{\mathrm{SD}}$ expression in the type II NB lineage gave normal adults at $25^{\circ} \mathrm{C}$. opa1 RNAi and marf RNAi expression in type II NB lineage when performed at a higher temperature of $29^{\circ} \mathrm{C}$ with pointed-Gal4 gave sluggish adults.

Mitochondria are tubular and uniformly distributed around the nucleus in type I NBs [31]. To characterize mitochondrial morphology in type II NBs, we performed super-resolution Stimulated Emission Depletion microscopy (STED) on mitochondria stained with antibody against ATP $\beta$ subunit of complex $V$ in the third instar larval brain. We observed thread-like mitochondria evenly distributed around the nucleus in control type II NBs (Figure 1B, S2A). We used pointed-Gal4, mCD8-GFP to identify the type II NBs using mCD8-GFP and deplete Opa1, Marf and Drp1. RNAi against mitochondrial fusion proteins Opa1 and Marf has been previously shown to deplete the corresponding mRNA and lead to mitochondrial fragmentation in electron microscopy studies [32-35]. We observed a distinct shift in mitochondrial morphology from a tubular organization to dispersed punctae on depletion of Opa1 and Marf using two different RNAi lines as compared to mcherry-RNAi controls, confirming the requirement of these proteins for mitochondrial fusion (Figure 1B, S2A). We quantified the numbers and area of optically 
resolvable mitochondria in STED images to estimate the mitochondrial size in each NB. NBs depleted of Opa1 and Marf showed a significant increase in mitochondrial numbers and decrease in mitochondrial area as compared to controls (Figure 1C-D, S2B-C). Interestingly, the extent of mitochondrial fragmentation was similar upon depletion of either Opa1 or Marf. Further, expression of Drp1 ${ }^{\mathrm{SD}}$ resulted in clustering of mitochondria on one side of the NB suggesting that mitochondria were fused (Figure 1B). Drp1 depletion led to a significant increase in mitochondrial area and decrease in mitochondrial numbers as compared to controls (Figure 1C-D). In summary, depletion of Opa1 and Marf led to mitochondrial fragmentation and depletion of Drp1 led mitochondrial fusion in the type II NBs.

\section{Mitochondrial fusion is required for differentiation in the type II NB lineage}

We checked the effect of knockdown of mitochondrial morphology proteins on NB number, polarity and differentiation. We depleted mitochondrial morphology proteins using worniu-Gal4 to estimate the numbers of type I and II NBs. The overall number of NBs was not changed upon depletion of Opa1, Marf and Drp1 (Figure S3A-B) and the type II number also remained unaffected (Figure S3C). Apico-basal distribution of polarity proteins Bazooka and Numb in metaphase is essential for fate determination in the lineage [26]. We found that the NBs did not show a defect in polarised distribution of Bazooka and Numb on depletion of Opa1, Marf or Drp1 (Figure S3D).

We identified cells in each type II NB lineage based on their specific molecular profile (Figure 1A). Dpn immunostaining was used to count mINPs (Figure 2A) while nuclear Pros immunostaining was used to count GMCs (Figure 2B) in type II NB lineage. Immature INPs lacked both Dpn and Pros. Immature INPs were similar in number in Opa1, Marf and Drp1 depleted type II NB lineages (Figure 2C). On the other hand, mINPs and GMCs were lowered on expression of two different RNAi lines against opa1 as compared to controls (Figure 2A,B,D,E, S2D-G). Marf depletion by different RNAi lines did not alter mINP numbers but reduced GMCs as compared to controls (Figure 2A,B,D,E, S2D-G). There was no change in mINPs and GMCs in each lineage 
178 in Drp1 ${ }^{\mathrm{SD}}$ expressing type II NBs (Figure 2A,B,D,E). The numbers of GMCs were also 179 reduced in the opa1 RNAi but not the marf depleted type I NB lineages (Figure S3E-F). 180 Loss of mitochondrial fusion in Opa1 and Marf mutants may give rise to defective INPs 181 thereby impairing differentiation in the type II NB lineage. to a greater extent compared to Marf, despite comparable disruption of mitochondrial morphology to fragmentation. Opa1 and Marf depletion may give rise to loss of cells in the lineage due to defects in the type II NB and mINP division. Depletion of mitochondrial fission protein Drp1 did not show any defects in differentiation. The phenotype of increased mitochondrial fragmentation and delay in NB division thereby leading to loss of differentiation has been previously noted on depletion of ETC components [23]. Apart from mitochondrial fusion, Opa1 is also involved in maintenance of cristae architecture [11,36-38]. It is therefore possible that a specific defect in mitochondrial inner membrane organization caused by Opa1 depletion in addition to loss of fusion is a cause for a loss in mINPs and GMCs in the type II NB 193 lineage.

\section{Depletion of mitochondrial fission protein Drp1 along with Opa1 or Marf shows}

\section{fused mitochondria and suppresses the defects in differentiation}

Mitochondrial fragmentation in opa1 and marf depleted cells requires the activity of mitochondrial fission protein Drp1 [30,32,39,40]. To alleviate the mitochondrial fragmentation defect seen in opa1 and marf knockdown, we made combinations of Drp1 $^{\mathrm{SD}}$;mRFP (data not shown), Drp1 ${ }^{\mathrm{SD}}$;opa1 RNAi and Drp1 ${ }^{\mathrm{SD}}$;marf RNAi and analyzed mitochondrial morphology. Unlike opa1 RNAi and marf RNAi (Figure 1), the $\operatorname{Drp}^{\mathrm{SD}}$;opa1 RNAi and Drp1 ${ }^{\mathrm{SD}}$;marf RNAi combinations showed clustered mitochondrial morphology. The mitochondrial cluster was more resolved as compared to Drp1 ${ }^{\mathrm{SD}}$ alone

204 and Drp1 ${ }^{\text {SD }}$, mRFP (data not shown), with a few punctate mitochondria appearing and a 205 small but significant decrease in average mitochondrial area in double mutants (Figure $2063 \mathrm{~A}-\mathrm{C})$. 
We analyzed these double mutants for differentiation of type II NB lineage by staining larval brains with Dpn and Pros. The numbers of Dpn positive mINPs increased in Drp1 ${ }^{\mathrm{SD}}$;opa1 RNAi as compared to opa1 alone and were not significantly different from controls (Figure 3D-E). mINPs were similar to controls in marf RNAi and Drp1 $^{\mathrm{SD}}$;marf RNAi mutants (Figure 3D-E). The Drp1 ${ }^{\mathrm{SD}}$;opa1 RNAi combination showed an increase in Pros positive GMCs but did not completely restore their numbers (Figure 3F-G). GMC numbers also increased in the Drp1 ${ }^{\mathrm{SD}}$;marf combination as compared to 214 marf RNAi (Figure 3F-G). In summary, the differentiation defect observed in opa1 and marf depletion was partially reversed on mitochondrial fusion by additional depletion of 216 Drp1. suppressed by additional depletion of Drp1 membrane potential and activity. Mitochondrial membrane potential (MMP) is a readout for mitochondrial quality and functionality [41]. We checked the effect of mitochondrial dynamics proteins depletion on MMP by using the potentiometric dye, Tetra-methylrhodamine methyl ester (TMRM) in vivo in living larval brains by live imaging. We estimated the relative fluorescence obtained from uptake of TMRM in mCD8-GFP marked type II NBs as compared to neighboring unmarked controls. We found a significant decrease in TMRM fluorescence and therefore MMP on depletion of Opa1 and Marf whereas in Drp1 ${ }^{\mathrm{SD}}$ expression did not affect the MMP (Figure 4A-B). Codepletion of Drp1 along with Opa1 or Marf RNAi suppressed the MMP defect showing that outer mitochondrial membrane fusion restores mitochondrial membrane potential (Figure 4A-B). the cell that could trigger a stress response. We have previously found that change in

234 ETC activity but not mitochondrial fusion obtained by Drp1 depletion causes ATP stress which further triggers phosphorylation of AMP activated protein kinase (pAMPK). 
237 pAMPK levels elevate in energy deprived conditions and act as an energy sensor inside 238 the cell [43]. To check whether ATP stress was seen in mitochondrial fusion mutant 239 NBs, we stained brains with antibodies against pAMPK. NB differentiation relies at least 240 in part on glycolytic metabolism for ATP and loss of ATP in ETC mutants is seen when 241 depleted of both glycolysis and ETC activity [22,23]. Therefore as a positive control for 242 reduction in glycolysis and induction of pAMPK we added 2-deoxy-glucose (2-DG), a 243 non-hydrolysable analogue of glucose to larval brains after dissection and observed a 244 significant increase in pAMPK levels throughout the brain. However, we did not observe 245 a change in pAMPK levels in opa1 and marf mutant type II NBs as compared to 246 neighboring NBs indicating that ATP stress similar to 2-DG treatment was not seen in 247 these mutants (Figure S4A-B). This shows that depletion of ATP as read out by 248 decrease in mitochondrial membrane potential in NBs was much lower as compared to 249 that seen when glycolysis was inhibited with 2-DG.

Another consequence of change in mitochondrial morphology is alteration in the levels of reactive oxygen species (ROS) $[44,45]$. We estimated ROS levels using

252 Dihydroxy ethidium (DHE) fluorescence in NBs as compared to neighboring cells.

253 Consistent with previous studies [34,46], we observed increased DHE fluorescence 254 indicating increased ROS on opa1 knockdown and Drp1 ${ }^{\text {SD }}$ expression (Figure S4C-D). 255 marf depletion however did not change the DHE fluorescence significantly (Figure S4C256 D). Since increased ROS was found in both opa1 and drp1 mutant NBs, and there was 257 no effect on differentiation on Drp1 ${ }^{\mathrm{SD}}$ expression (Figure 2), we conclude that ROS is 258 unlikely to play a significant role in differentiation in type II NBs. We further checked if a 259 general increase in ROS can affect development. We found that in type I and II NBs 260 expressing a mutant of human Superoxide dismutase (hSOD) [30,47], ROS was 261 increased significantly (Figure S4C-D). However, hSOD expression in all NBs did not 262 affect viability of flies and adults emerged similar to controls leading us to conclude that 263 it did not impact differentiation and functionality of neurons (data not shown). and loss of MMP of mitochondria in NBs, Opa1 depletion showed a specific decrease in 266 mINPs as compared to Marf knockdown. Opa1 oligomerization leads to stabilization of 
cristae and localization of cytochrome $\mathrm{c}$ in a packed manner in cristae. Increase in cytochrome c occurs when Opa1 oligomerization is decreased and this correlates with loose cristae organization [36]. We visualized the distribution of cytochrome $\mathrm{c}$ in opa1 and marf mutant NBs. Increase in cytochrome c was seen in opa1 RNAi as compared to marf RNAi and controls (Figure 4C-D). This suggests that loosening of cristae architecture potentially causes specific spread of the cytochrome c signal on Opa1 depletion. Altogether, loss of MMP and increased cytochrome c in type II NBs deficient

274 of opa1 is suggestive of disruption of inner mitochondrial membrane architecture and activity in addition to fusion leading to loss of differentiation. mitochondrial fusion on additional depletion of Drp1

The decreased lineage size observed in opa1 and marf mutant type II NBs may result from apoptosis or lowered proliferation rates of each NB thereby reducing the numbers of INPs and GMCs in each lineage. Increased cytochrome C and ROS in opa1 depletion suggests that type II NB differentiation could be decreased by apoptosis in the lineage [45,48-50]. For probing apoptosis, we assessed the levels of cleaved caspase in opa1 mutants. Cleaved caspase staining was not elevated in opa1 mutant lineages and was similar to controls (Figure S4E). Selectively marking apoptotic nuclei with the Terminal deoxynucleotidyl transferase (TdT) dUTP Nick-End Labeling (TUNEL) assay did not show any significant difference between Opa1 mutant NBs and control. We validated this assay by using UAS-hid expression as a positive control for apoptosis induction (Figure S4F-G). Therefore, cell death is not responsible for the differentiation defect in opa1 mutant type II NBs.

mINPs divide asymmetrically to produce GMCs in the type II NB lineage. We analyzed the numbers of cells in mitosis in each type II NB lineage by staining for phospho-histone $3(\mathrm{pH} 3)$ antibody. We found a significant reduction of $\mathrm{pH} 3$ positive

294 cells in the type II lineage in opa1 mutants while marf and drp1 depletion did not show 295 any significant change as compared to controls (Figure 5A-B). The numbers of pH3 
296 positive cells were increased in the Drp1 ${ }^{\mathrm{SD}}$;opa1 RNAi combination as compared to 297 opa1 RNAi and controls. The Drp1 ${ }^{\mathrm{SD}}$;marf RNAi combination also showed significant 298 increase in pH3 positive cells per lineage as compared to controls indicating an 299 increase in proliferation of mINPs in this combination (Figure 5A-B). The loss of pH3 300 positive cells showed that Opa1 depletion decreased the rate of mINP proliferation in 301 the type II NB. This defect of loss of proliferation in Opa1 mutants was suppressed on 302 outer mitochondrial fusion in Drp1 mutants.

Notch signaling drives differentiation and fused mitochondrial morphology in the type II NB lineage

Notch signaling regulates numbers of NBs and their differentiation in the type II lineage in the Drosophila third instar larval brain [28,29,51]. Notch signaling is activated in type II NBs and suppressed in INPs. Notch signaling is again activated in the mINPs and GMCs in the lineage. Loss of Notch signaling leads to transformation of the type II NB to the type I NB with absence of Dpn positive mINPs. We overexpressed full length Notch $\left(\right.$ Notch $^{\mathrm{FL}}$ ) to increase Notch signaling and notch RNAi to decrease Notch signaling in the type II NB lineage. As documented previously [52], we observed an increased number of Dpn positive NBs on Notch ${ }^{\mathrm{FL}}$ expression and a decreased number

314 of type II NBs in notch RNAi expressing type II NBs. However, depletion of 315 mitochondrial morphology proteins did not change the numbers of NBs (Figure S3A-C).

316 Therefore, we conclude that the Notch signaling needed for maintenance of type II NB 317 numbers was not defective in these mutants. We further analyzed the numbers of Dpn 318 positive mINPs in lineages of remaining type II NBs that were present on notch RNAi 319 expression. As expected, we observed decreased Dpn positive mINPs in notch RNAi while they remained unaffected in Notch ${ }^{\mathrm{FL}}$ expressing type II NB lineage (Figure 6A-B).

Since Notch signaling regulated differentiation within each type II NB lineage and 322 loss of mitochondrial fusion proteins led to loss of differentiation in the type II NB 323 lineage, we assessed if Notch signaling regulated mitochondrial morphology in type II 324 NBs. STED microscopy revealed thread like mitochondria evenly distributed around the 
325 nucleus in type II NBs (Figure 6C). Interestingly, overexpression of the Notch ${ }^{\mathrm{FL}}$ and

326 Nintra in type II NBs led to clustering of mitochondria on one side of the nucleus (Figure

$3276 \mathrm{C})$. Overexpression of Notch ${ }^{\mathrm{FL}}$ led to an increase in the average area of mitochondria

328 and decrease in mitochondrial numbers per cell as compared to control NBs (Figure 6D-

329 E). Contrarily, Notch downregulation by expression of notch RNAi led to an increase in

330 fragmented mitochondria as marked by a decrease in average size of optically

331 resolvable puncta and increase in numbers per cell. Likewise, depletion of $\mathrm{Su}(\mathrm{H})$ in type

332 II NBs also resulted in fragmented mitochondria (Figure 6C-E). These data show that

333 Notch activation via the canonical pathway through $\mathrm{Su}(\mathrm{H})$ leads to mitochondrial

334 clustering and fusion whereas Notch depletion results in fragmented mitochondria.

Mitochondrial fusion induces differentiation on Notch depletion in the type II NB lineage

Our data together shows that mitochondrial morphology is tubular in type II NBs and fusion of mitochondria alone did not give a differentiation defect but was able to suppress the differentiation defect in opa1 mutant NBs. We further tested whether mitochondrial fusion would drive differentiation in type II NBs depleted of Notch. We combined drp1 mutants to generate fused mitochondria along with notch RNAi and expressed this combination in the type II NB lineage (Figure 7A). The numbers of type II NBs remained less than controls in the notch RNAi, Drp1 ${ }^{\mathrm{SD}}$ combination and were similar to notch RNAi (Figure 7B). This observation further confirms that mitochondrial fusion does not affect Notch signaling in the type II NB. The loss of mINPs (Figure 7A,C) and GMCs (Figure 7D,E) was suppressed in each type II NB lineage and the 348 numbers of mINPs and GMCs was closer to controls (Figure 7A,C,D,E). These results show that fused mitochondrial morphology can alleviate Notch signalling defects to drive differentiation in the type II NB lineage.

To check whether fused mitochondrial morphology is essential for Notch

352 signaling mediated NB proliferation, we depleted Opa1 and Marf along with Notch ${ }^{\mathrm{FL}}$

353 overexpression and found that the numbers of the NBs were significantly reduced as 
354 compared to Notch ${ }^{\mathrm{FL}}$ alone (Figure 7F-G). Overexpression of the intracellular domain of 355 Notch gave rise to an increase in size of brain lobes. The brain lobe size was reduced 356 on depletion of Opa1 and Marf on overexpression of Notch intra (Figure S5A). The NB 357 numbers were reduced in the Nintra; opa1 RNAi and Nintra; marf RNAi combination 358 (Figure S5B-C). As expected, the clustered mitochondrial morphology seen on overexpression of Nintra (Figure 6C) was also not seen on depletion of Opa1 and Marf in the Nintra background (Figure S5D). We therefore conclude that increased Notch signaling along with fused mitochondrial architecture leads to NB proliferation.

In summary, our results show that Notch signaling increased mitochondrial fusion and mitochondrial fusion was important for differentiation in the type II NB lineage. This is also consistent with the requirement of mitochondrial fusion in NB tumors induced in brat and numb mutants, since depletion of mitochondrial fusion and not mitochondrial fission abrogates the tumor phenotype in brat and numb mutant NBs [24]. In summary our results show that mitochondrial fusion controlled by Opa1 and Marf regulates Notch signaling driven differentiation in the Drosophila type II NB lineage.

\section{Discussion}

Recent evidence shows that maintenance of mitochondrial architecture is critical for cell fate determination [53-55]. Depletion of ETC components in Drosophila NBs

373 leads to mitochondrial fragmentation and loss of differentiation and cancer progression

374 [22-24]. Here we show that mitochondrial fusion proteins, Opa1 and Marf affect

375 differentiation in the type II NB lineage (Figure 8A-B). Opa1 and Marf gave equivalent

376 fragmentation and loss of mitochondrial activity in the type II NB. However, decreased

377 Opa1 led to loss of both mINP and GMCs whereas decreased Marf led to loss of only

378 GMCs in the type II lineage. Interestingly, mitochondrial outer membrane fusion

379 ameliorated the defects in mitochondrial activity and differentiation caused by Opa1 and 380 Marf depletion. Notch signaling regulated fused mitochondrial morphology in the type II 381 NB and mitochondrial fusion in Notch depleted type II NB lineages led to suppression of 382 differentiation defects. Here, we discuss our results in the following contexts: 1] the 
mechanisms by which Notch signaling gives rise to fused mitochondrial morphology, 2] the role of the mitochondrial morphology in mediating Notch signaling and 3] the role of mitochondrial fusion in differentiation in NBs.

\section{Regulation of mitochondrial fusion by Notch signaling}

At the heart of the discussion on interaction between Notch signaling and mitochondrial morphology, lies an analysis of how Notch signaling regulates mitochondrial fusion and activity. Loss of $s u(H)$ also showed fragmented mitochondria similar to downregulation of Notch. In addition, mitochondrial fusion alleviated the differentiation defects seen in type II NBs depleted of Notch. Since the action of both $\mathrm{NICD}$ and $\mathrm{Su}(\mathrm{H})$ is needed in the nucleus to activate targets downstream of Notch signaling, it is possible that Notch regulates fused mitochondrial morphology through the canonical pathway by enhancing expression of fusion genes opa1 and marf or regulating activity of Opa1 and Marf by post translational modification and reducing expression or activity of Drp1. Notch signaling may also regulate mitochondrial activity by elevating components of the ETC. Recent studies show that NB tumors arising in brat and numb RNAi, which also have elevated Notch signaling, show increased

400 transcription of Opa1 and Marf thereby causing mitochondrial fusion and increased 401 oxidative metabolism resulting from an increase in expression of oxidative 402 phosphorylation enzymes [24,29]. This is likely to occur due to increased Notch activity 403 or due to the change of fate of cells in the type II NB lineage.

\section{Impact of mitochondrial morphology on Notch signaling}

407 though increased Notch signaling by pointed-Gal4 favored mitochondrial fusion in the 408 Dpn positive NBs, loss of Opa1 and Marf did not affect the numbers of type I and type II 409 NBs. This evidence suggests that mitochondrial fusion is not needed during Notch 410 mediated formation of NBs during the embryonic stages. We found that proliferation of 
411 mINPs depended upon Notch signaling driven mitochondrial fusion. Even though the

412 immature INP numbers in mitochondrial mutants did not change in mitochondrial fusion

413 mutants, it is possible that mitochondrial fusion is needed to produce healthy INPs that

414 will mature, proliferate and differentiate to form GMCs. Further, a decrease in GMCs on

415 Opa1 and Marf depletion is possible because of defects in division of the mINPs. Loss

416 of mitochondrial fusion led to abrogation of mitochondrial activity. How might loss of

417 mitochondrial fusion and activity affect differentiation in the type II NB lineage?

418 Mitochondrial morphology changes may have an effect on the fate of the cell by

419 regulating key metabolites (Choi et al. 2020; Galloway and Yoon 2013). Mitochondrial

420 pyruvate carrier (MPC) in the inner mitochondrial membrane is involved in transport of

421 pyruvate from cytoplasm to the mitochondrial matrix where pyruvate undergoes

422 oxidation via tricarboxylic acid (TCA) cycle (McCommis and Finck 2015). MPC depletion

423 in the intestinal stem cells (ISC) results in increased ISC proliferation and loss of

424 differentiation (Schell et al. 2017). We observed severe differentiation defects upon loss

425 of Opa1 in NB lineage. It is possible that the defects in the inner mitochondrial

426 membrane due to Opa1 loss affect pyruvate transport into the mitochondrial which

427 further affect NB differentiation. It will be interesting to check whether pyruvate

428 metabolism is affected in the mitochondrial morphology mutants.

$429 \quad$ Our preliminary analysis shows that Opa1 mutant type II NBs show a loss of

430 Notch receptors on the plasma membrane and increase in endosomes in the type II NB

431 and the lineage (data not shown). Numb regulates inhibition of Notch signaling in INPs

432 [29]. It is possible that sustained activity of Numb in INPs and mINPs generated from

433 NB proliferation leads to a decrease in Notch signaling in the mINPs. Future

434 experiments on the defects on Notch receptor trafficking and Numb distribution in type II

435 NBs will reveal a mechanistic link between mitochondrial morphology, activity and Notch 436 signaling.

Notch signaling may also be affected by interaction between mitochondrial

438 morphology and other upstream signaling pathways. Interestingly, our finding that Notch

439 signaling maintains fused mitochondria in NBs is in striking contrast to recent literature

440 in other systems where fused mitochondrial morphology has been correlated with loss 
441 of notch activity. In triple negative breast cancer (TNBC) Notch signaling enhances

442 mitochondrial fission via drp1 [56]. In Drosophila ovarian posterior follicle cells,

443 mitochondrial fusion induces an increase in mitochondrial membrane potential and loss

444 of Notch signaling [18,19]. Loss of opa1 in cardiomyocytes leads to decrease in

445 differentiation due in enhanced Notch processivity [20]. It is interesting to speculate the

446 reasons for observing tissue specific differences in the requirement of mitochondrial

447 fusion for Notch signaling in differentiation. We had previously found that EGFR

448 signaling regulates fragmented morphology for appropriate Notch signaling in

449 Drosophila follicle cells $[18,19]$. It is likely that fragmented mitochondrial morphology

450 leads to an interaction between EGFR and Notch signaling pathways in follicle cells and

451 possibly other cell types such as cardiomyocytes and TNBCs where Notch signaling

452 increase occurs only on loss of mitochondrial membrane potential or fragmentation.

454 Role of mitochondrial fusion in Notch driven differentiation in NBs

Mitochondrial fusion is coincident with elaborate cristae organization, increased activity and increased oxidative phosphorylation [38,57,58]. Clustered mitochondria on one side of the nucleus produced on the depletion of Drp1, did not show any defect on NB differentiation suggesting that Drp1 is dispensable for NB formation and differentiation. Interestingly, Drp1 loss driven mitochondrial hyperfusion could suppress the mitochondrial activity and differentiation defects in Opa1 and Marf mutant type II NBs. Mitochondrial fragmentation and loss of mitochondrial membrane potential is likely 462 to cause a reduction in ATP [59]. However, ATP depletion has been reported only on 463 combined loss of oxidative phosphorylation and glycolysis and brat RNAi driven tumors 464 rely on NAD+ metabolism rather than ATP synthesis [22-24]. Since we did not see any 465 ATP stress in Opa1 and Marf depleted type II NBs, it is interesting to speculate that 466 other mitochondrial functions dependent upon mitochondrial activity from oxidative 467 phosphorylation are important for type II NB differentiation. Indeed mitochondrial activity 468 may give rise to changes in calcium buffering and key metabolites [60] thereby affecting 469 Notch signaling in NBs and lineage cells depleted of Opa1 and Marf. 
Since depletion of inner mitochondrial membrane protein Opa1 affected differentiation to a greater extent as compared to Marf, it is possible that organization of

472 the mitochondrial ETC complexes and cristae architecture in addition to fusion are

473 crucial for type II NB proliferation and differentiation. Organization of the cristae

474 architecture independent of oxidative phosphorylation has been previously shown to be

475 important for Drosophila germ line stem cell differentiation [61]. Fusion of the outer

476 mitochondrial membrane may restore the inner membrane organisation and increase

477 mitochondrial activity needed in type II NB proliferation and differentiation. Recent

478 evidence suggests that neurodegeneration defects caused by depletion of oxidative

479 phosphorylation in Purkinje neurons can be completely rescued by mitochondrial fusion

480 produced by overexpression of Mfn [62]. NBs deficient of the mitochondrial ETC lead to

481 fragmentation [23] and it will be interesting to probe if fusion of mitochondria in ETC

482 mutants will also mitigate the differentiation defect.

In summary, we find a distinct role for mitochondrial fusion in regulation of Notch

484 signaling in type II NB differentiation. Future studies on the mechanistic link between

485 mitochondrial activity, change in metabolite status and Notch signaling in diverse

486 contexts will give further insight into the interaction between Notch signaling and

487 mitochondrial morphology in a tissue specific manner. Our studies motivate an analysis

488 of mechanisms that regulate the interaction between mitochondrial fusion or inner

489 membrane architecture and signaling during development and differentiation at large.

\section{Acknowledgements}

492 We thank the RR lab members for discussions on this project and feedback on the 493 manuscript. We thank the Drosophila facility and microscopy facility at IISER, Pune, 494 India for support throughout this project. DD thanks IISER, Pune, India for his graduate 495 fellowship. RR thanks DBT and IISER Pune for funding. 


\section{Fly genetics}

499 Fly crosses were performed in standard cornmeal agar medium and raised $29{ }^{\circ} \mathrm{C}$. The 500 following fly lines were used in this study: pnt>Gal4,UAS-mCD8-GFP (Jurgen Knoblich, 501 IMP, Vienna, Austria), elav>Gal4, prospero>Gal4, inscuteable>Gal4, scabrous>Gal4, 502 worniu>Gal4, opa1 RNAi (Bloomington stock number BL32358), opa1 RNAi (Ming Guo, 503 UCLA), marf RNAi (Ming Guo, UCLA, [33]), marf RNAi (BL31157 ), opa1 RNAi2 504 (BL67159), marf RNAi2 (BL67158, [63]), drp1 RNAi (BL51483), drp1 RNAi

505 (VDRC44155), Drp1 ${ }^{\text {S193D }}$ (Drp1 ${ }^{\text {SD }}$, GTPase domain mutant, acts as a dominant 506 negative, made in the Richa Rikhy lab), notch RNAi (BL31383), su(H)RNAi (BL67928), 507 UAS-Notch ${ }^{\mathrm{FL}}$ (BL52309) and UAS-Nintra (LS Shashidhara, IISER, Pune, India), hSOD1 508 mutant (BL33607), mCherry RNAi (BL35785). Drp1 ${ }^{\mathrm{SD}}$; opa1 RNAi , Drp1 ${ }^{\mathrm{SD}}$; marf RNAi, notch RNAi /CyOGFP; opa1 RNAi /TM3SerGFP, notch RNAi /CyOGFP; marfRNAi /TM3SerGFP lines were generated using standard genetic crosses. worniu>Gal4,UASmCD8-GFP (wor-Gal4) was used to express transgenes in all the larval NBs in the third instar larval brain and pointed-Gal4 (pnt-Gal4) was used to express transgenes in the

513 type II NBs in the larval brain.

\section{Immunostaining of larval brain}

515 Wandering third instar larvae were dissected in Schneider's medium and immediately

516 fixed in 4\% PFA solution for 25 minutes at room temperature (RT). The brains were 517 washed subsequently with 1 X PBS with $0.1 \%$ Triton X-100 (PBST) for 30 minutes at $518 \mathrm{RT}$. They were blocked with 1\% BSA for $1 \mathrm{hr}$ at RT. The brains were stained with the 519 appropriate primary antibody overnight at $4^{\circ} \mathrm{C}$. They were then washed 3 times with $520 \quad 0.1 \%$ PBST (first wash for 20 minutes and remaining for 10 minutes each). An 521 appropriate fluorescently coupled secondary antibody was added for $1 \mathrm{hr}$ at RT followed 522 by three washes with $0.1 \%$ PBST (first wash for 20 min and remaining for 10 min each) 523 and mounted in Slow-Fade Gold (Molecular Probes).

524 The following dilutions were used for the primary antibodies: chicken anti-GFP (1:1000, 525 Invitrogen), anti- ATPß (1:200, Abcam), anti- Deadpan (1:150, Abcam), anti- Prospero 526 (1:25, DSHB), anti- Miranda (1:600, Abcam), anti-Cytochrome C (1:200, Cell Signaling), 
527 anti-Elav (1:100, DSHB), anti-cleaved Caspase3 (1:100, Cell Signaling), anti-

528 phosphohistone 3 (1:100, Invitrogen), anti-phosphoAMPK (1:200, Invitrogen). Hoechst

529 (1:1000, Molecular Probes) was used to label DNA. Fluorescently coupled secondary

530 antibodies (Molecular Probes): anti-Chicken 488, anti-Rat 568/633/647, anti-Rabbit 568,

531 anti-Mouse 568/633 were used in 1:1000 dilution.

\section{DHE uptake for live imaging of ROS}

533 Dissected third instar larval brains were treated with Dihydroethidium (DHE) (1:1000,

534 Molecular Probes) in Schneider's medium for 15 minutes at RT and then washed with

535 Schneider's medium for $10 \mathrm{~min}$. Brains were mounted in LabTek chambers containing

536 Schneider's medium and imaged immediately using Zeiss LSM 710 with a 63x/1.4NA oil

537 objective using a DPSS $(561 \mathrm{~nm})$ laser and dihydroethidium-1 filter settings in the

538 Zeiss2010 software. The laser power, acquisition speed, frame size and gain were kept

539 the same for both control and mutant. The laser power and gain were adjusted to keep

540 the range of acquisition between 0-255 on an 8-BIT scale.

\section{$541 \quad$ 2-Deoxy glucose treatment}

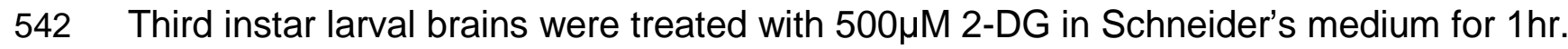

543 Control and treated brains were processed for pAMPK immunostaining as mentioned

544 above.

\section{Mitochondrial membrane potential estimation with TMRM in live brains}

546 Third instar larval brains were dissected in Schneider's medium. Then they were treated

547 with Tetramethylrhodamine, methyl ester (TMRM) (100nM, Thermo fisher Scientific) for

54830 mins at room temperature. Treated brains were mounted in a LabTek chamber

549 containing Schneider's medium and imaged immediately using Zeiss LSM710 with a

$55063 x / 1.4 N A$ oil objective. A DPSS $(561 \mathrm{~nm})$ laser and RFP/TRITC filter was used for the 551 detection of TMRM signal.

\section{TUNEL assay for detection of apoptotic cells}


553 Third instar wandering larvae were dissected in Schneider's medium and fixed in 4\%

554 PFA for 25 mins followed by washing with $0.1 \%$ PBST for 20 mins at RT. Brains are then washed twice with 1XPBS for 2 min. The terminal transferase (TdT) reaction was

556 performed as follows: First brains were treated with TdT reaction buffer and incubated

557 for 10min followed by treatment of freshly made TdT reaction buffer cocktail (TdT

558 reaction buffer, 5-Ethynyl-dUTP, TdT) for $60 \mathrm{~min}$ at $37^{\circ} \mathrm{C}$ and at 500rpm. After the TdT

559 reaction, brains were washed twice with 3\% BSA at RT for 5 min. Then Click-iT reaction

560 was performed by adding click iT reaction cocktail (Click-iT reaction buffer, Click-iT

561 reaction buffer additive) for $30 \mathrm{~min}$ at $\mathrm{RT}$. At this step samples were protected from light.

562 Brains were washed with 3\% BSA twice for 5 min after removing Click-iT reaction buffer

563 cocktail and then incubated with Hoescht (1:1000) for DNA staining followed by washing

564 with 1 XPBS for 10 min. Samples mounted in Slow-Fade Gold (Molecular probes) and

565 subsequently imaged using Zeiss LSM710 with a 40x/1.4NA oil objective and DPSS

566 laser $(561 \mathrm{~nm})$. Induction of apoptosis by UAS-Hid line driven by pointed Gal4 was used

567 as positive control. TUNEL positive nuclei were counted and plotted using GraphPad

568 Prism 5 software.

569

Microscopy and Image acquisition

Imaging of fixed samples using a confocal microscope

572 Confocal microscopy of fixed samples was done at room temperature using LSM710 or

573 LSM780 inverted microscope (Carl Zeiss, Inc. and IISER Pune microscopy facility) with

574 a Plan apochromat 40x 1.4NA and 63x 1.4NA oil objective. Images were acquired using

575 the Zen2010 software at $1024 \times 1024$ pixels with an averaging of 4 and acquisition speed

576 7. Fluorescence intensity was kept within 255 on an 8-bit scale. Following lasers were

577 used for the excitation of different fluorophores during fixed sample imaging: Diode laser

578 for Hoescht, Argon laser line at 488nm for Alexa Fluor 488, DPSS laser for Alexa Fluor

579568 , HeNe (633nm) for Alexa Fluor 633 and Alexa Fluor 647. The representative image

580 for each type II lineage in the figures is shown from the center of the lineage and

581 comprises the maximum number of cells in the lineage. 
Stimulated Emission-Depletion (STED) microscopy for imaging mitochondrial morphology

585 Super-resolution microscopy was done for visualizing mitochondrial morphology within type II NBs using the Leica TCS SP8 STED 3X Nanoscope with a 100x/1.4NA oil

587 objective. Images were acquired using the LasX software at $1024 \times 1024$ pixels to keep 588 pixel size $20-25 \mathrm{~nm}$, an averaging of 4 , an acquisition speed of 200 and a zoom of 4.5.

589 The Alexa Fluor 488 and 568 were excited with Argon $488 \mathrm{~nm}$ and Diode $561 \mathrm{~nm}$ lasers 590 respectively and emission were collected with hybrid detectors for GFP and 591 mitochondria labeled with ATP $\beta$ antibody respectively. The $561 \mathrm{~nm}$ excitation laser with 592 the $775 \mathrm{~nm}$ depletion laser was used for stimulated emission-depletion for visualizing 593 mitochondria by super resolution. Fluorescence intensity was kept within 255 on an 8-bit 594 scale using the LUT mode to avoid over-saturated pixels.

Image analysis and statistics

\section{Neuroblasts number analysis}

599 NBs in a single hemisphere were counted by using Miranda as a NB marker across 600 different mitochondrial dynamics mutants and compared with controls. Type II NBs were 601 counted by using mCD8-GFP positive lineages expressed under pointed Gal4. Non602 parametric student t-test was performed for statistical analysis.

\section{ROS intensity analysis}

605 Fluorescence intensity of DHE uptake in control and mutant type II NB (mCD8-GFP 606 positive) along with their neighboring NB (mCD8-GFP positive) were quantified by 607 drawing a region of interest using a free hand tool in ImageJ. Ratios of DHE 608 fluorescence intensity of GFP positive NB to a neighboring GFP negative NB present in 609 the same optical plane were plotted using GraphPad Prism software. Non-parametric 610 student t-test was performed for statistical analysis

611 pH3 Analysis 
$612 \mathrm{pH}$ positive cells were counted in each type II NB lineage for control and mutant brains

613 and plotted using GraphPad Prism software. Non-parametric student t-test was

614 performed for statistical analysis.

Deadpan and Prospero quantification for mINPs and GMCs

616 Immature INPs were identified and quantified as Dpn- and Pros-cells in each type II NB

617 lineage labelled with mCD8-GFP and Dpn and Pros. Dpn positive mINPs were counted

618 in all sections in each type II NB lineage in control and mutant brains. Numbers of

619 mINPs were plotted using GraphPad Prism software and non- parametric student-t-test

620 was performed for statistical analysis. Nuclear Pros containing GMCs were counted in

621 the type II NB lineage. For Pros analysis in each brain hemisphere for larval NBs of the

622 type I and type II lineage, the maximum intensity optical plane was selected from

623 anterior and posterior regions. We obtained the average area for each Pros positive

624 nucleus from 5 GMCs in each sample. The total Pros positive area was determined by

625 using the threshold tool in ImageJ. To extract the number of Pros positive GMCs, we

626 divided the total area by average area of a single GMC.

\section{TMRM analysis}

628 TMRM intensities were computed in the entire type II NB in control, opa1 RNAi and marf 629 RNAi expressing brains and in the clustered mitochondria in Drp1 ${ }^{\mathrm{SD}}$, Drp1 ${ }^{\mathrm{SD}}$;opa1 RNAi

630 and Drp1 ${ }^{\mathrm{SD}}$;marf RNAi expressing brains using ImageJ. Average intensity was

631 computed from the GFP positive type II NB and expressed as a ratio to the intensity

632 seen in the neighboring control GFP negative NB. Relative intensities were then plotted

633 using GraphPad Prism software.

\section{PAMPK analysis}

635 Average pAMPK intensities in control and mutant GFP positive type II NB and 636 neighboring GFP negative NBs were measured using ImageJ software. pAMPK

637 fluorescence was plotted as a ratio to the neighboring control cells by using GraphPad

638 Prism software. Larval brain treatment of 2-DG showed an overall increase in antibody 
639 fluorescence and the intensity in the GFP positive type II NB was used as positive

640 control to induction of pAMPK.

\section{Mitochondrial number and area analysis}

642 Control and mutant brains were immunostained with ATP $\beta$ antibody. A qualitative 643 observation of mitochondrial distribution as tubular, clustered and dispersed was made

644 by visually observing NBs in various genotypes. Quantitative measurements for size

645 and number of mitochondria were also done by using thresholding tool in imageJ

646 software. A single optical plane was selected approximately in the middle of the type II

647 NB (section with the highest diameter) where the nucleus was prominently visible and

648 the density of the mitochondria was high. All mitochondrial particles which were above

649 size cut off of $0.12 \mu \mathrm{m}^{2}$ were analyzed for size. To clearly resolve mitochondrial

650 particles in different genotypes we used a watershading tool from ImageJ.

\section{TUNEL analysis}

652 TUNEL positive nuclei per central brain region or type II NB lineage were counted in 653 lobes of control and opa1 RNAi and plotted using GraphPad Prism software. We 654 induced apoptosis by expressing UAS-Hid with pnt Gal4 as a positive control for TUNEL 655 assay.

Figure legends

Figure 1: Mitochondrial morphology protein depletion leads to altered mitochondrial distribution in neuroblasts

661 A: Schematic of larval CNS (left) containing the central brain (CB) lobes and ventral 662 nerve cord (VNC) and type I (blue) and type II NB (purple) distribution and lineages of 663 type I (blue, right) and type II NB (purple, right). The type I NB lineage has Dpn 664 (magenta nuclei) positive NBs and Pros (green nuclei) positive GMCs. The type II NB 665 lineage has Dpn positive NBs (magenta nuclei), Dpn negative and Pros negative 666 immature INPs (black and white), Dpn positive mINPs (magenta nuclei) and Pros 
667 positive GMCs (green nuclei). Dpn positive NBs are clearly distinguishable as larger

668 cells as compared to Dpn positive mINPs in each type II NB lineage.

669 B-D: Mitochondrial morphology and distribution in type II NBs (white dotted line,

670 magnified area shown in the panel on the right) stained with ATP $\beta$ (red) antibody using

671 STED super resolution microscopy is shown in representative images with zoomed

672 inset in the right panel (B). mCherry RNAi (100\% tubular, 32 NBs, 8 brains), opa1 RNAi

673 (100\% fragmented, 46,8), marf RNAi (100\% fragmented, 45,8), Drp1 ${ }^{\text {SD }}$ (85\% clustered,

674 80,10). Average mitochondrial area quantification from type II NBs (C) in mCherry RNAi

675 (7 type II NBs, 3 brains), opa1 RNAi $(9,3)$, marf RNAi $(11,3)$, Drp1 ${ }^{\mathrm{SD}}(10,3)$.

676 Mitochondrial number quantification in mCherry RNAi $(7,3)$, opa1 RNAi $(9,3)$, marf RNAi

$677(11,3), \operatorname{Drp1}^{S D}(10,3)(D)$. Scale bar- $5 \mu \mathrm{m}$

678 C-D: Graphs show mean \pm sd. Statistical analysis is done using an unpaired t-test. **

$679 \mathrm{p}<0.01,{ }^{* *}-\mathrm{p}<0.001$.

680

681

682 II NB lineage

683 A-B: Representative confocal images of the type II NB lineage showing that Dpn

684 positive mINPs (red, yellow arrows) are reduced in pnt-Gal4, UAS-mCD8-GFP driven

685 opa1 RNAi (A) and Pros (red, yellow arrows) positive GMCs (B) are lowered in opa1

686 RNAi and marf RNAi expressing type II NBs

687 C: Quantification of immature INPs (Dpn and Pros negative) per type II NB lineage.

688 mCherry RNAi (8 type II NB lineages, 4 Brains), opa1 RNAi $(14,5)$, marf RNAi $(11,3)$,

$689 \operatorname{Drp1}^{\mathrm{SD}}(6,3)$.

690 D: Quantification of Dpn positive mINPs (yellow arrows) per type II NB lineage in

691 mCherry RNAi ( $\mathrm{n}=22$ type II NB lineages, 4 brains), opa1 RNAi $(21,6)$, marf RNAi

$692(13,8), \operatorname{Drp1}^{\mathrm{SD}}(28,10)$. 
693 E: Quantification of Pros positive GMCs (yellow arrows point to nuclear Pros) per type II 694 NB lineage (E) of mCherry RNAi (18 type II NB lineages,5 brains), opa1 RNAi $(18,5)$, 695 marf RNAi $(19,5)$, Drp1 ${ }^{\mathrm{SD}}(26,9)$. Scale bar- 10um

696 C, D, E: Graphs show mean \pm sd. Statistical analysis is done using an unpaired t-test. 697 ns=non-significant, ${ }^{* *}-p<0.01,{ }^{* * *}-p<0.001$.

Figure 3: Drp1 depletion in opa1 RNAi and marf RNAi expressing type II NB lineages leads to a suppression of the differentiation defect.

A-C: Representative images with zoomed inset in the right panel of type II NBs (white dotted line) containing pnt-Gal4, mCD8-GFP (green) stained for mitochondrial morphology with ATP $($ red) and imaged using STED (A). pnt-Gal4, UAS-mCD8-GFP with Drp1 ${ }^{\mathrm{SD}}$ (85\% clustered, 80 NBs, 10 Brains), Drp1 ${ }^{\mathrm{SD}}$;opa1 RNAi (85\% clustered, 96,12), Drp1 ${ }^{\mathrm{SD}}$;marf RNAi (86\% clustered, 96,12). Quantification of average mitochondrial area (B) in type II NB of $\operatorname{Drp} 1^{S D}(6,3)$, Drp1 ${ }^{S D}$;opa1 RNAi $(7,4)$, $\operatorname{Drp1}^{\mathrm{SD}}$; marf RNAi $(7,4)$. Quantification of mitochondrial numbers $(\mathrm{C})$ in type II NB of

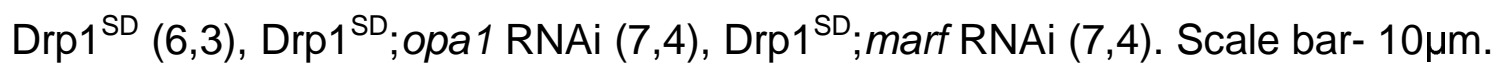

711 NB lineages, 16 brains), Drp1 ${ }^{\text {SD }}(28,10)$, opa1 RNAi $(20,8)$, Drp1 ${ }^{\text {SD }}$;opa1 RNAi $(8,6)$,

712 marf RNAi $(13,8)$, Drp1 ${ }^{\mathrm{SD}}$; marf RNAi $(35,8)$. Scale bar- $10 \mu \mathrm{m}$.

713 F-G: Type II NBs lineages (yellow dotted line) showing expression of mCD8-GFP

714 (green) and Pros (red, yellow arrows point to nuclear Pros) (F). Quantification of Dpn

715 positive mINPs $(\mathrm{G})$ in control (29 NB lineages, 18 brains), Drp1 ${ }^{\mathrm{SD}}(26,8)$, opa1 RNAi

716 (20,8), Drp1 ${ }^{\mathrm{SD}}$; opa1 RNAi $(26,8)$, marf RNAi $(14,8)$, Drp1 ${ }^{\mathrm{SD}}$; marf RNAi $(33,8)$. Scale bar-

$717 \quad 10 \mu \mathrm{m}$

718 B,C,E \& G: Graphs show mean \pm sd. Comparative analysis was done by using unpaired 719 t-test. ns- non significant, ${ }^{* *}-p<0.01,{ }^{* * *}-p<0.001$ 
Figure 4: Co-depletion of Drp1 in Opa1 or Marf depleted type II NBs rescues the reduced mitochondrial membrane potential.

A-B: Representative images showing decreased TMRM intensity in opa1 RNAi and marf RNAi expressing type II NBs. pnt-Gal4, UAS-mCD8-GFP control (17 type II NBs, 8 brains), $\operatorname{Drp}^{\mathrm{SD}}(9,5)$, opa1 RNAi $(16,5)$, Drp1 ${ }^{\mathrm{SD}}$; opa1 RNAi $(11,4)$, marf RNAi $(11,5)$,

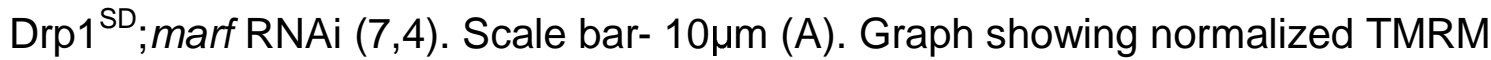
intensities in control (17 type II NBs, 8 brains), Drp1 ${ }^{\mathrm{SD}}(9,5)$, opa1 RNAi $(16,5)$, Drp1 $^{\mathrm{SD}}$;opa1 RNAi 7,4), marf RNAi $(11,5)$, Drp1 ${ }^{\mathrm{SD}}$;marf RNAi $(6,4)(\mathrm{B})$. normalised with control (D). Control (8 brains), opa1 RNAi (7 brains), marf RNAi (7 brains).

B,D: Graphs show mean \pm sd. Comparative analysis was done by using unpaired t-test. ns- non significant, ${ }^{* *}-\mathrm{p}<0.01,{ }^{* * *}-\mathrm{p}<0.001$ inhibition of Drp1

A-B: Representative confocal images of type II NB lineages show loss of pH3 positive cells on opa1 RNAi expressed with pnt-Gal4, UAS-mCD8-GFP in the type II NB lineage (A). Quantification of pH3 positive cells (B) in control (28 NB lineages, 6 brains), Drp1 ${ }^{\mathrm{SD}}$

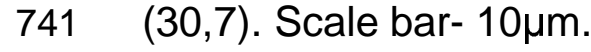
non significant, ${ }^{*}-p<0.1,{ }^{* *}-p<0.01,{ }^{* * *}-p<0.001$ 
A-B: Type II NB lineages stained for mCD8-GFP (green) and Dpn (red, yellow arrows) show reduced mINPs in Notch downregulation (D). Analysis of mINP numbers in type II NB lineages (E) of pnt-Gal4, UAS-mCD8-GFP control (16 NB lineages, 4 brains), or with Notch $^{\mathrm{FL}}$ (Notch full length) $(16,8)$, notch RNAi $(5,5)$. Scale bar-10 $\mathrm{mm}$.

751 C-E: Type II NBs (mCD8-GFP, green, yellow dotted line) showing Notch signaling 752 mediated regulation of mitochondrial morphology (red) by ATP $\beta$ antibody using STED 753 microscopy (A). Control (100\% tubular, 75 NBs, 22 Brains), Notch ${ }^{\mathrm{FL}}$ (68\% clustered, 754 103,16), Nintra (76\% clustered, 58,6), notch RNAi (50\% fragmented, 30,14), su(H) RNAi 755 (80\% fragmented, 23,12). Average mitochondrial area quantification (B) in Control (6 756 NBs,3 brains), Notch ${ }^{\mathrm{FL}}(10,5)$, Nintra $(6,4)$, notch RNAi $(6,4)$, su(h) RNAi $(7,4)$.

757 Mitochondrial number quantification (C) in control $(6,3), \operatorname{Notch}^{\mathrm{FL}}(10,5)$, Nintra $(6,4)$, 758 notch RNAi $(6,4)$, su(H) RNAi $(7,4)$. Scale bar- $5 \mu \mathrm{m}$.

759 B, D \& E: Graphs show mean \pm sd. Statistical analysis was done by using unpaired t760 test. ns- non significant, ${ }^{*}-p<0.1,{ }^{* *}-p<0.01,{ }^{* * *}-p<0.001$

Figure 7: Drp1 depletion alleviates the differentiation defect seen in notch RNAi expressing type II NB lineages.

764 A-C: Type II lineages (pnt-Gal4, mCD8-GFP, green) showing Dpn positive INPs (red, 765 yellow arrows) (A). Quantification of number of type II NBs (B) in control (16 Brains), $766 \operatorname{Drp1}^{\mathrm{SD}}(10)$, notch RNAi (9), Drp1 ${ }^{\mathrm{SD}}$;notch RNAi (5). Quantification of Dpn positive 767 mINPs (C, yellow arrows)) in control (23 NB lineages, 16 brains), Drp1 ${ }^{\mathrm{SD}}(28,10)$, notch 768 RNAi (54,9), Drp1 ${ }^{\mathrm{SD}}$;notch RNAi $(25,5)$. Scale bar- 10 $\mu \mathrm{m}$

769 D-E: Type II lineages (pnt-Gal4, mCD8-GFP, green) showing Pros positive INPs (red, 770 yellow arrows) (D, yellow arrows). Quantification of Pros positive GMCs (E) in control

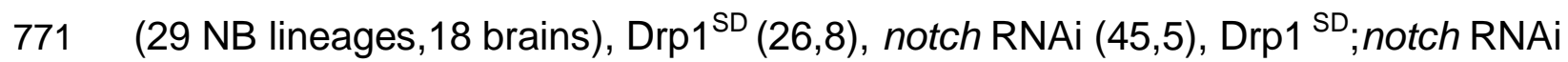

772 (33,5). Scale bar- 10um 
773 F-G: Larval brain lobes show suppression of Notch mediated NB hyper proliferation on

774 depletion of opa1 and marf $(\mathrm{F})$. Quantification of NB number (G) in pnt-Gal4, UAS-

775 mCD8-GFP control (14 lobes), Notch ${ }^{\mathrm{FL}}$ (11), Notch ${ }^{\mathrm{FL}}$; opa1 RNAi (9), Notch ${ }^{\mathrm{FL}}$; marf RNAi

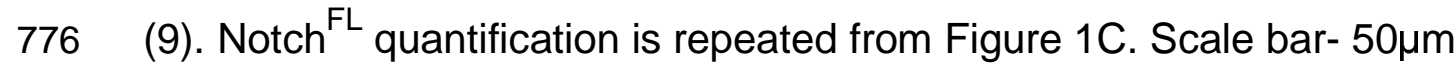

777 B, C, E \& G: Graphs show mean \pm sd. Statistical analysis was done by using unpaired t778 test. ns- non significant, ${ }^{* * *}-\mathrm{p}<0.001$.

Figure 8: Schematic summary for regulation of type II NB differentiation by fused mitochondrial morphology

782 Mitochondria are maintained in a relatively tubular morphology in type II NBs in the 783 presence of canonical Notch signaling. Mitochondrial fusion protein Opa1 is essential 784 for NB differentiation (A). Mitochondrial fusion protein Marf is also essential for 785 formation of GMCs. Depletion of mitochondrial fusion protein Opa1 in type II NBs leads

786 to formation of fragmented mitochondria with lowered mitochondrial membrane potential 787 which can potentially result in the production of defective immature INPs which further 788 fails to differentiate into mINP and GMCs (B).

790 Supplementary figure legends:

791 Figure S1: Table showing analysis of mitochondrial morphology protein 792 knockdown with different neuronal Gal4s.

793 Various Gal4 drivers were crossed with mutants of mitochondrial morphology genes at 79425 or $29^{\circ} \mathrm{C}$ and lethality or behavioral phenotype was recorded in the adult. worniu795 Gal4 (wor-Gal4), inscuteable-Gal4, prospero-Gal4 and scabrous-Gal4 expresses the 796 Gal4 in all NBs, pointed-Gal4 (pnt-Gal4) expresses in type II NBs and elav-Gal4 797 expresses in neurons. Adult flies from crosses with wor-Gal4 $\left(25^{\circ} \mathrm{C}\right)$ and pnt-Gal4 $(29$ $798{ }^{\circ} \mathrm{C}$ ) with opa1 RNAi and marf RNAi were sluggish and the numbers obtained were at the 799 expected frequency, no lethality was seen at the pupal stage. elav-Gal4 crosses gave 
800 lethality and few adults emerged. The opa1 RNAi BL32358 and opa1 RNAi2 BL67158

801 BL gave stronger phenotypes as compared to opa1 RNAi Ming Guo lab with inscuteable-Gal4, prospero-Gal4, elav-Gal4 and scabrous-Gal4. The marf RNAi Ming

803 Guo lab and marf RNAi2 BL67159 gave stronger phenotypes as compared to marf 804 RNAi BL31157 with inscuteable-gal4, prospero-Gal4 and elav-Gal4. We chose opa1 RNAi BL32358, opa1 RNAi2 BL67158, marf RNAi Ming Guo lab and marf RNAi BL67159 for further analysis. The Drp1 RNAi from VDRC did not show phenotypes and 807 the Drp1 (BL51483) gave inconsistent results, hence we used a lab generated GTPase 808 domain mutant Drp1 ${ }^{\mathrm{SD}}$ for further analysis.

809 Figure S2: Depletion of mitochondrial fusion proteins Opa1 and Marf result in mitochondrial fragmentation and loss of type II NB differentiation

811 A-C:Mitochondrial morphology and distribution in type II NBs (white dotted line,

812 magnified area shown in the panel on the right) stained with ATP $\beta$ (red) antibody using

813 STED super resolution microscopy is shown in representative images with zoomed

814 inset in the right panel (A). Control (100\% tubular, 75 NBs, 22 brains), opa1 RNAi2

815 (100\% fragmented, 14,4), marf RNAi2 (100\% fragmented, 14,4). Average mitochondrial 816 area quantification from type II NBs (B) in control (10 type II NBs, 4 brains), opa1 RNAi2 $817(8,4)$, marf RNAi2 $(5,3)$. Mitochondrial number quantification in control $(10,4)$, opa1 818 RNAi2 $(8,4)$, marf RNAi2 $(5,3)(C)$. Scale bar- $5 \mu \mathrm{m}$

819 D-E:Type II NB lineages (yellow dotted line) showing expression of mCD8-GFP (green) 820 and Dpn (red, yellow arrows) (D). Quantification of Dpn positive mINPs (E) in control (20 821 NB lineages, 5 brains), opa1 RNAi2 $(19,4)$, marf RNAi2 $(15,4)$. Scale bar- $10 \mu \mathrm{m}$.

822 F-G:Type II lineages (pnt-Gal4, mCD8-GFP, green) showing Pros positive INPs (red, 823 yellow arrows) (D, yellow arrows). Quantification of Pros positive GMCs (E) in control 824 (28 NB lineages, 8 brains), opa1 RNAi2 $(19,4)$, marf RNAi2 $(18,4)$. Scale bar- 10 $\mu$ m.

825 B-C, E, G: Graphs show mean \pm sd. Statistical analysis is done using an unpaired t-test. 826 ns- non significant, ${ }^{* *}-p<0.01,{ }^{* * *}-p<0.001$. 
Figure S3: NB polarity and number are unaffected by knockdown of mitochondrial

\section{morphology proteins}

829 A-B: Representative confocal images of larval brain lobes stained for Miranda (red)

830 show no change in NB number (A). Expression of opa1 RNAi, marf RNAi and Drp1 ${ }^{\mathrm{SD}}$

831 mutant were done by wor-Gal4. Quantification of NB number in larval brain lobes (B) of

832 control (13 lobes, 13 brains), opa1 RNAi $(10,10)$, marf RNAi $(10,10)$, Drp1 ${ }^{\text {SD }}(10,10)$.

833 Scale bar- $50 \mu \mathrm{m}$

834 C: Number of type II NBs and their progenies per lineage in control, opa1 RNAi, marf 835 RNAi, Drp1 ${ }^{\text {SD }}$ ( $n=30$ type II NB lineages, 15 brains each) .

836 D: Representative images showing apical and basal localization of Bazooka and Numb

837 respectively in control and mitochondrial dynamics mutants. Scale bar- $10 \mu \mathrm{m}$

838 E-F: Representative images of brain lobes showing reduced Pros positive GMC 839 population in wor-Gal4 opa1 RNAi (E). Analysis of Pros positive GMCs $(\mathrm{F})$ in control 840 (10 lobes, 10 brains), opa1 RNAi $(11,11)$, marf RNAi $(7,7)$, Drp1 ${ }^{\mathrm{SD}}(11,11)$.Scale bar$84150 \mu \mathrm{m}$

842 B, C \& F: Graphs show mean \pm sd. Statistical analysis was done by using unpaired t843 test. ns- non significant, ${ }^{* * *}$ - $p<0.001$ Figure S4: Depletion of Opa1 does not cause ATP stress and apoptosis in type II NB lineages

847 A-B: Representative images of type II NBs expressing mitochondrial fusion mutants

848 along with pnt-Gal4, UAS-mCD8-GFP did not show change in levels of pAMPK (pAMPK 849 fluorescence is shown as rainbow scale) (A). Controls, mutants and 2-DG treated brains 850 were imaged for PAMPK fluorescence at the same time under the same imaging 851 conditions. Type II NBs are marked by white dotted lines while lineages are marked by 852 expression of mCD8-GFP (green). Analysis of pAMPK intensity normalised with 
853 neighboring control cells (B). Control (41 NBs, 14 brains), opa1 RNAi $(33,8)$, marf RNAi $854(23,8)$, DMSO $(76,8), 2-D G(91,9)$.

855 C-D: Representative images showing increased levels of ROS (rainbow scale) in type II 856 NB (position marked by white dotted line) using pnt-Gal4, UAS-mCD8-GFP with

857 mitochondrial dynamics mutants $(\mathrm{C})$. Analysis of relative DHE fluorescence as a ratio to 858 neighboring cells (D) in type II NBs, control (22 NBs,6 Brains), opa1 RNAi (17,8), marf $859 \operatorname{RNAi}(17,8), \operatorname{Drp1}^{\mathrm{SD}}(9,5)$, hSOD1 mutant $(13,3)$.

860 E-G: Representative images of type II NB lineages (pnt-Gal4, UAS-mCD8-GFP, green) 861 stained for cleaved caspase and shown in heatmap in opa1 RNAi (E) control (10 NB 862 lineages,6 Brains), opa1 RNAi $(9,6)$. Fluorescence confocal images of brain 863 hemispheres showing no significant change in TUNEL positive nuclei (red) (F) in opa1 864 RNAi expressed in all NBs with wor-Gal4. Expression of UAS-hid shows a significant 865 increase in TUNEL positive cells when expressed with pnt-Gal4, mCD8-GFP in the type 866 II NB lineage. Quantification of TUNEL positive nuclei (G) in worsWT(3 brains), wors 867 opa1 RNAi (4), pnt> UAS-hid reaper (5). Statistical analysis was performed using 868 unpaired t-test. ns- non significant, ${ }^{* *}-\mathrm{p}<0.0001$

869 B, D \& G: Graphs show mean \pm sd. Statistical analysis was done by using unpaired t870 test. ns- non significant, ${ }^{* *}-p<0.01^{* * *}-p<0.001$

Figure S5: Nintra overexpression driven NB proliferation is partially reversed by additional depletion of Opa1 or Marf.

874 A-C: Larval brain lobes (CD8-GFP, green) (A) increase in size on Nintra overexpression 875 which is rescued by opa1 RNAi and marf RNAi expression. Representative images of 876 larval brain hemisphere containing mCD8-GFP (green, A, B) DNA (blue, B) and Dpn 877 (red, B) showing partial rescue of NB hyperproliferation in Nintra expression by opa1 878 RNAi and marf RNAi (B). Nintra (8 lobes, 8 brains), Nintra;opa1 RNAi (10,10), Nintra; 879 marf RNAi $(10,10)$. Scale bar- 50 $\mathrm{mm}$. Quantification of lobe size (C) in control (8 brains), 880 Nintra (12), Nintra;opa1 RNAi (10), Nintra; marf RNAi (12). C: Graph shows mean \pm sd. 
881 Statistical analysis was performed using unpaired t-test. ${ }^{*}-p<0.05,{ }^{* * *}-p<0.001$. Scale 882 bar- $200 \mu \mathrm{m}$.

883 D: Representative superresolution STED images of mitochondria showing clustered 884 morphology in Nintra mutant expressed with pnt-Gal4, UAS-mCD8-GFP and punctate 885 appearance in opa1 and marf RNAi expressing type II NBs in the Nintra background. 886 Nintra (76\% tubular, 58 Neuroblasts, 6 Brains), Nintra;opa1 RNAi (100\%, 95, 20), 887 Nintra;marf RNAi (100\%, 98, 14). Scale bar- 10um

\section{References}

1. Xu X, Duan S, Yi F, Ocampo A, Liu G-H, Izpisua Belmonte JC. Mitochondrial regulation in pluripotent stem cells. Cell Metab. 2013;18: 325-332.

2. Prigione A, Fauler B, Lurz R, Lehrach $\mathrm{H}$, Adjaye J. The senescence-related mitochondrial/oxidative stress pathway is repressed in human induced pluripotent stem cells. Stem Cells. 2010;28: 721-733.

3. Suhr ST, Chang EA, Tjong J, Alcasid N, Perkins GA, Goissis MD, et al. Mitochondrial rejuvenation after induced pluripotency. PLoS One. 2010;5: e14095.

4. Schell JC, Wisidagama DR, Bensard C, Zhao H, Wei P, Tanner J, et al. Control of intestinal stem cell function and proliferation by mitochondrial pyruvate metabolism. Nat Cell Biol. 2017;19: 1027-1036.

5. Ito K, Suda T. Metabolic requirements for the maintenance of self-renewing stem

6. Tilokani L, Nagashima S, Paupe V, Prudent J. Mitochondrial dynamics: overview of molecular mechanisms. Essays Biochem. 2018;62: 341-360.

7. Westermann B. Molecular machinery of mitochondrial fusion and fission. J Biol Chem. 2008;283: 13501-13505.

8. Hoppins S, Lackner L, Nunnari J. The machines that divide and fuse mitochondria. Annu Rev Biochem. 2007;76: 751-780.

9. Meeusen S, DeVay R, Block J, Cassidy-Stone A, Wayson S, McCaffery JM, et al. Mitochondrial inner-membrane fusion and crista maintenance requires the dynamin-related GTPase Mgm1. Cell. 2006;127: 383-395.

911 10. Olichon A, Guillou E, Delettre C, Landes T, Arnauné-Pelloquin L, Emorine LJ, et al. 
Mitochondrial dynamics and disease, OPA1. Biochim Biophys Acta. 2006;1763:

914 500-509.

11. Frezza C, Cipolat S, Martins de Brito O, Micaroni M, Beznoussenko GV, Rudka T, et al. OPA1 controls apoptotic cristae remodeling independently from mitochondrial fusion. Cell. 2006;126: 177-189.

12. Sara Cogliati, Jose A Enriquez, Luca Scorrano. Mitochondrial Cristae: Where Beauty Meets Functionality. - PubMed - NCBI. Trends in Biochemical Science. 2016;41: 261-273.

13. Rambold AS, Kostelecky B, Lippincott-Schwartz J. Fuse or die: Shaping mitochondrial fate during starvation. Commun Integr Biol. 2011;4: 752-754.

14. Gomes LC, Di Benedetto G, Scorrano L. During autophagy mitochondria elongate, are spared from degradation and sustain cell viability. Nat Cell Biol. 2011;13: 589598.

15. Rolland SG, Motori E, Memar N, Hench J, Frank S, Winklhofer KF, et al. Impaired complex IV activity in response to loss of LRPPRC function can be compensated by mitochondrial hyperfusion. Proc Natl Acad Sci U S A. 2013;110: E2967-76.

16. Tondera $D$, Grandemange S, Jourdain A. SLP $\square 2$ is required for stress $\square$ induced mitochondrial hyperfusion. EMBO J. 2009. Available: https://www.embopress.org/doi/abs/10.1038/emboj.2009.89

17. Yamamoto S, Charng W-L, Bellen HJ. Endocytosis and intracellular trafficking of Notch and its ligands. Curr Top Dev Biol. 2010;92: 165-200.

18. Tomer D, Chippalkatti R, Mitra K, Rikhy R. ERK regulates mitochondrial membrane potential in fission deficient Drosophila follicle cells during differentiation. Dev Biol. 2018;434: 48-62.

19. Mitra K, Rikhy R, Lilly M, Lippincott-Schwartz J. DRP1-dependent mitochondrial fission initiates follicle cell differentiation during Drosophila oogenesis. J Cell Biol. 2012;197: 487-497.

20. Kasahara A, Cipolat S, Chen Y, Dorn GW 2nd, Scorrano L. Mitochondrial fusion directs cardiomyocyte differentiation via calcineurin and Notch signaling. Science. 2013;342: 734-737.

21. Khacho M, Clark A, Svoboda DS, Azzi J, MacLaurin JG, Meghaizel C, et al. Mitochondrial Dynamics Impacts Stem Cell Identity and Fate Decisions by Regulating a Nuclear Transcriptional Program. Cell Stem Cell. 2016;19: 232-247.

22. Homem CCF, Steinmann V, Burkard TR, Jais A, Esterbauer H, Knoblich JA. Ecdysone and mediator change energy metabolism to terminate proliferation in Drosophila neural stem cells. Cell. 2014;158: 874-888. 
23. van den Ameele $\mathrm{J}$, Brand $\mathrm{A}$. Neural stem cell temporal patterning and brain tumour growth rely on oxidative phosphorylation. eLife. 2019. doi:10.7554/elife.47887

24. Bonnay F, Veloso A, Steinmann V, Köcher T, Abdusselamoglu MD, Bajaj S, et al. Oxidative Metabolism Drives Immortalization of Neural Stem Cells during Tumorigenesis. Cell. 2020;182: 1490-1507.e19.

25. Gallaud E, Pham T, Cabernard C. Drosophila melanogaster Neuroblasts: A Model for Asymmetric Stem Cell Divisions. In: Tassan J-P, Kubiak JZ, editors. Asymmetric Cell Division in Development, Differentiation and Cancer. Cham: Springer International Publishing; 2017. pp. 183-210.

26. Homem CCF, Knoblich JA. Drosophila neuroblasts: a model for stem cell biology. Development. 2012;139: 4297-4310.

27. Bayraktar OA, Boone JQ, Drummond ML, Doe CQ. Drosophila type II neuroblast lineages keep Prospero levels low to generate large clones that contribute to the adult brain central complex. Neural Dev. 2010;5: 26.

28. Li X, Xie Y, Zhu S. Notch maintains Drosophila type II neuroblasts by suppressing expression of the Fez transcription factor Earmuff. Development. 2016;143: 25112521.

29. Bowman SK, Rolland V, Betschinger J, Kinsey KA, Emery G, Knoblich JA. The tumor suppressors Brat and Numb regulate transit-amplifying neuroblast lineages in Drosophila. Dev Cell. 2008;14: 535-546.

30. Chowdhary S, Madan S, Tomer D, Mavrakis M, Rikhy R. Mitochondrial morphology and activity regulate furrow ingression and contractile ring dynamics in Drosophila cellularization. Mol Biol Cell. 2020; mbcE20030177.

31. Sen A, Damm VT, Cox RT. Drosophila clueless is highly expressed in larval neuroblasts, affects mitochondrial localization and suppresses mitochondrial oxidative damage. PLoS One. 2013;8: e54283.

32. Trevisan T, Pendin D, Montagna A, Bova S, Ghelli AM, Daga A. Manipulation of Mitochondria Dynamics Reveals Separate Roles for Form and Function in Mitochondria Distribution. Cell Rep. 2018;23: 1742-1753.

33. Deng H, Dodson MW, Huang H, Guo M. The Parkinson's disease genes pink1 and parkin promote mitochondrial fission and/or inhibit fusion in Drosophila. Proc Natl Acad Sci U S A. 2008;105: 14503-14508.

34. Macchi M, El Fissi N, Tufi R, Bentobji M, Liévens J-C, Miguel Martins L, et al. The Drosophila inner-membrane protein PMI controls crista biogenesis and mitochondrial diameter. J Cell Sci. 2013;126: 814-824.

35. Son JM, Sarsour EH, Kakkerla Balaraju A, Fussell J, Kalen AL, Wagner BA, et al. 
Mitofusin 1 and optic atrophy 1 shift metabolism to mitochondrial respiration during aging. Aging Cell. 2017;16: 1136-1145.

36. Patten DA, Wong J, Khacho M, Soubannier V, Mailloux RJ, Pilon-Larose K, et al. OPA1-dependent cristae modulation is essential for cellular adaptation to metabolic demand. EMBO J. 2014;33: 2676-2691.

37. Quintana-Cabrera R, Quirin C, Glytsou C, Corrado M, Urbani A, Pellattiero A, et al. The cristae modulator Optic atrophy 1 requires mitochondrial ATP synthase oligomers to safeguard mitochondrial function. Nat Commun. 2018;9: 3399.

38. Cogliati S, Enriquez JA, Scorrano L. Mitochondrial Cristae: Where Beauty Meets Functionality. Trends Biochem Sci. 2016;41: 261-273.

39. Otera $\mathrm{H}$, Miyata $\mathrm{N}$, Kuge $\mathrm{O}$, Mihara K. Drp1-dependent mitochondrial fission via MiD49/51 is essential for apoptotic cristae remodeling. J Cell Biol. 2016;212: 531544.

40. Deng H, Takashima S, Paul M, Guo M, Hartenstein V. Mitochondrial dynamics regulates Drosophila intestinal stem cell differentiation. Cell Death Discov. 2018;4: 17.

41. Zorova LD, Popkov VA, Plotnikov EY, Silachev DN, Pevzner IB, Jankauskas SS, et al. Mitochondrial membrane potential. Anal Biochem. 2018;552: 50-59.

42. Chowdhary S, Tomer D, Dubal D, Sambre D, Rikhy R. Analysis of mitochondrial organization and function in the Drosophila blastoderm embryo. Sci Rep. 2017;7: 5502.

43. Grahame Hardie D. AMP-activated/SNF1 protein kinases: conserved guardians of cellular energy. Nat Rev Mol Cell Biol. 2007;8: 774.

44. Ježek J, Cooper KF, Strich R. Reactive Oxygen Species and Mitochondrial Dynamics: The Yin and Yang of Mitochondrial Dysfunction and Cancer Progression. Antioxid Redox Signal. 2018;7. doi:10.3390/antiox7010013

45. H. -U. Simon H, Haj-Yehia A, Levi-Schaffer F. Role of reactive oxygen species (ROS) in apoptosis induction. Apoptosis. 2000;5: 415-418.

46. Tang S, Le PK, Tse S, Wallace DC, Huang T. Heterozygous Mutation of Opa1 in Drosophila Shortens Lifespan Mediated through Increased Reactive Oxygen Species Production. PLoS One. 2009;4: e4492.

47. Watson MR, Lagow RD, Xu K, Zhang B, Bonini NM. A drosophila model for amyotrophic lateral sclerosis reveals motor neuron damage by human SOD1. J Biol Chem. 2008;283: 24972-24981.

48. Ott M, Robertson JD, Gogvadze V, Zhivotovsky B, Orrenius S. Cytochrome c 
release from mitochondria proceeds by a two-step process. Proc Natl Acad Sci U S A. 2002;99: 1259-1263.

1021

1022

1023

1024

1025

1026

1027

1028

1029

1030

1031

1032

1033

1034

1035

1036

1037

1038

1039

1040

1041

1042

1043

1044

1045

1046

1047

1048

1049

1050

1051

1052

49. Liu K, Shu D, Song N, Gai Z, Yuan Y, Li J, et al. The role of cytochrome c on apoptosis induced by Anagrapha falcifera multiple nuclear polyhedrosis virus in insect Spodoptera litura cells. PLoS One. 2012;7: e40877.

50. Redza-Dutordoir M, Averill-Bates DA. Activation of apoptosis signalling pathways by reactive oxygen species. Biochim Biophys Acta. 2016;1863: 2977-2992.

51. Wang H, Somers GW, Bashirullah A, Heberlein U, Yu F, Chia W. Aurora-A acts as a tumor suppressor and regulates self-renewal of Drosophila neuroblasts. Genes Dev. 2006;20: 3453-3463.

52. Lee K-S, Wu Z, Song Y, Mitra SS, Feroze AH, Cheshier SH, et al. Roles of PINK1, mTORC2, and mitochondria in preserving brain tumor-forming stem cells in a noncanonical Notch signaling pathway. Genes Dev. 2013;27: 2642-2647.

53. Zhang $\mathrm{H}$, Menzies KJ, Auwerx J. The role of mitochondria in stem cell fate and aging. Development. 2018;145. doi:10.1242/dev.143420

54. Ma K, Chen G, Li W, Kepp O, Zhu Y, Chen Q. Mitophagy, Mitochondrial Homeostasis, and Cell Fate. Front Cell Dev Biol. 2020;8: 3184.

55. Bahat A, Gross A. Mitochondrial plasticity in cell fate regulation. J Biol Chem. 2019;294: 13852-13863.

56. Chen L, Zhang J, Lyu Z, Chen Y, Ji X, Cao H, et al. Positive feedback loop between mitochondrial fission and Notch signaling promotes survivin-mediated survival of TNBC cells. Cell Death Dis. 2018;9: 1050.

57. Westermann B. Bioenergetic role of mitochondrial fusion and fission. Biochim Biophys Acta. 2012;1817: 1833-1838.

58. Yao C-H, Wang R, Wang Y, Kung C-P, Weber JD, Patti GJ. Mitochondrial fusion supports increased oxidative phosphorylation during cell proliferation. Elife. 2019;8. doi:10.7554/eLife.41351

59. Chen $\mathrm{H}$, Chomyn $\mathrm{A}$, Chan DC. Disruption of fusion results in mitochondrial heterogeneity and dysfunction. J Biol Chem. 2005;280: 26185-26192.

60. Parekh $\mathrm{AB}$. Mitochondrial regulation of intracellular $\mathrm{Ca} 2+$ signaling: more than just simple Ca2+ buffers. News Physiol Sci. 2003;18: 252-256.

61. Teixeira FK, Sanchez CG, Hurd TR, Seifert JRK, Czech B, Preall JB, et al. ATP synthase promotes germ cell differentiation independent of oxidative phosphorylation. Nat Cell Biol. 2015;17: 689-696. 
bioRxiv preprint doi: https://doi.org/10.1101/2021.01 08.425832; this version posted January 8,2021 . The copyright holder for this preprint (which was not certified by peer review) is the author/funder, who has granted bioRxiv a license to display the preprint in perpetuity. It is made available under aCC-BY-NC 4.0 International license.

1053 62. Motori E, Atanassov I, Kochan SMV, Folz-Donahue K, Sakthivelu V, Giavalisco P, 1054 1055 et al. Neuronal metabolic rewiring promotes resilience to neurodegeneration caused by mitochondrial dysfunction. Sci Adv. 2020;6: eaba8271.

1056 63. Sandoval H, Yao C-K, Chen K, Jaiswal M, Donti T, Lin YQ, et al. Mitochondrial fusion but not fission regulates larval growth and synaptic development through steroid hormone production. Elife. 2014;3. doi:10.7554/eLife.03558 
A

Optic Lobe

$C B$

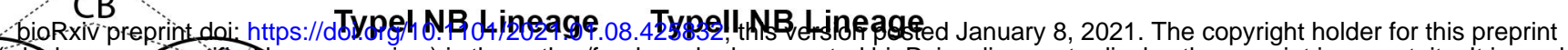
(wition was not certifified peer review) is the author/funder, who has granted bioRxiv a license to display the preprint in perpetuity. It is made

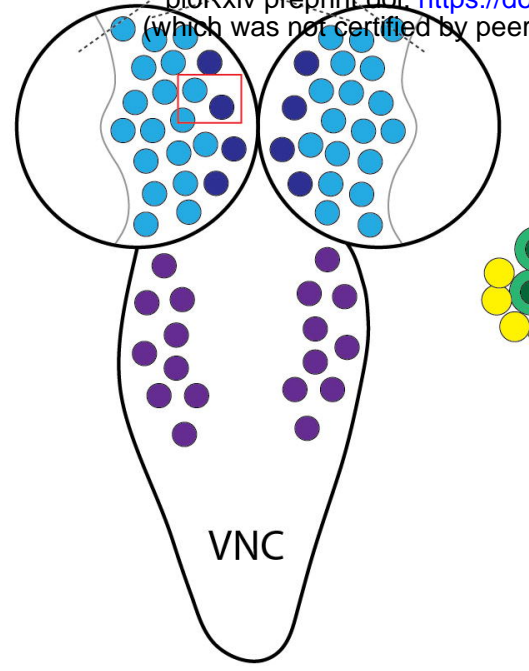
available under aCC-BY-NC 4.0 International license.

Typel NB (Deadpan+ve) Typell NB (Deadpan+ve)

B

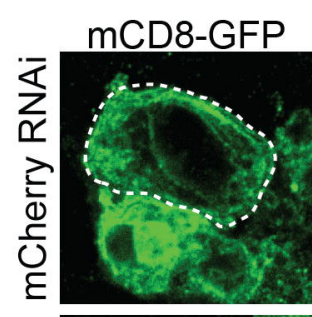

¿
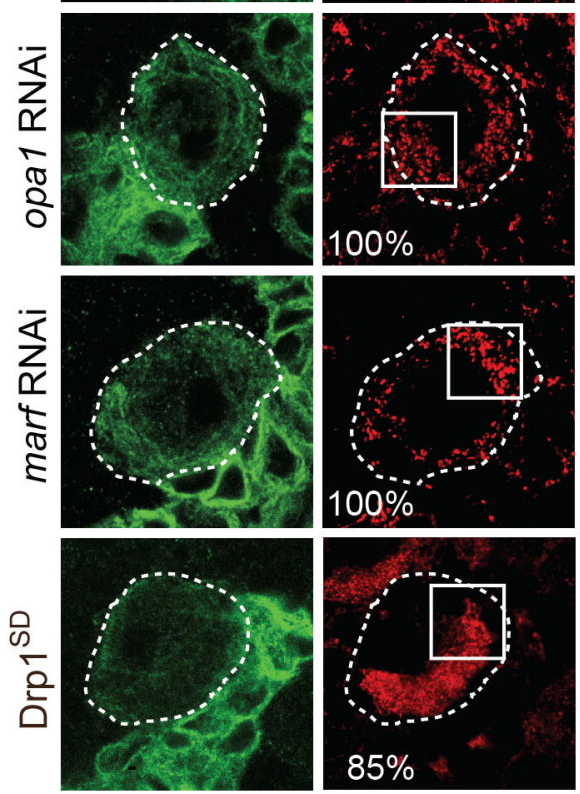

ATPB
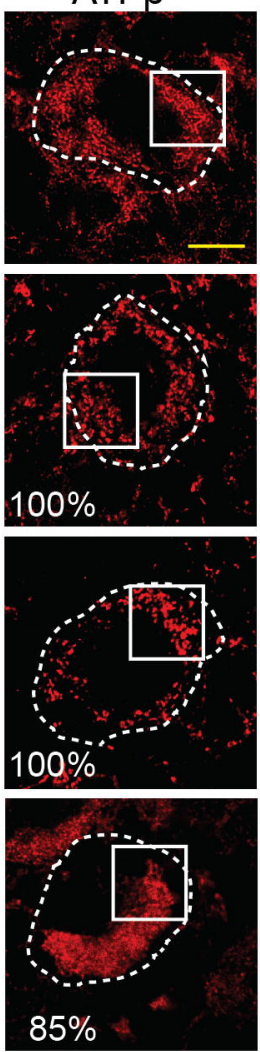

Inset
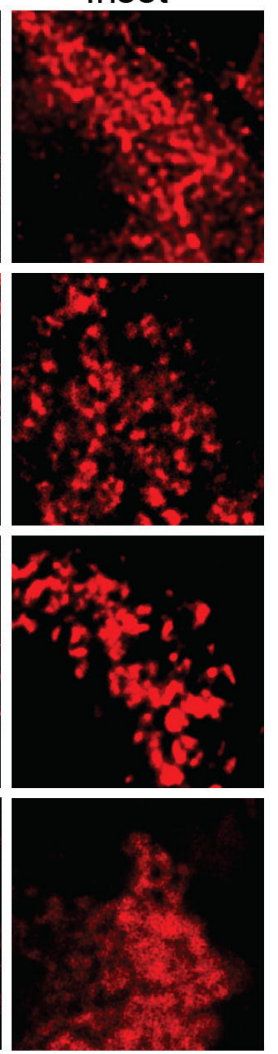

C
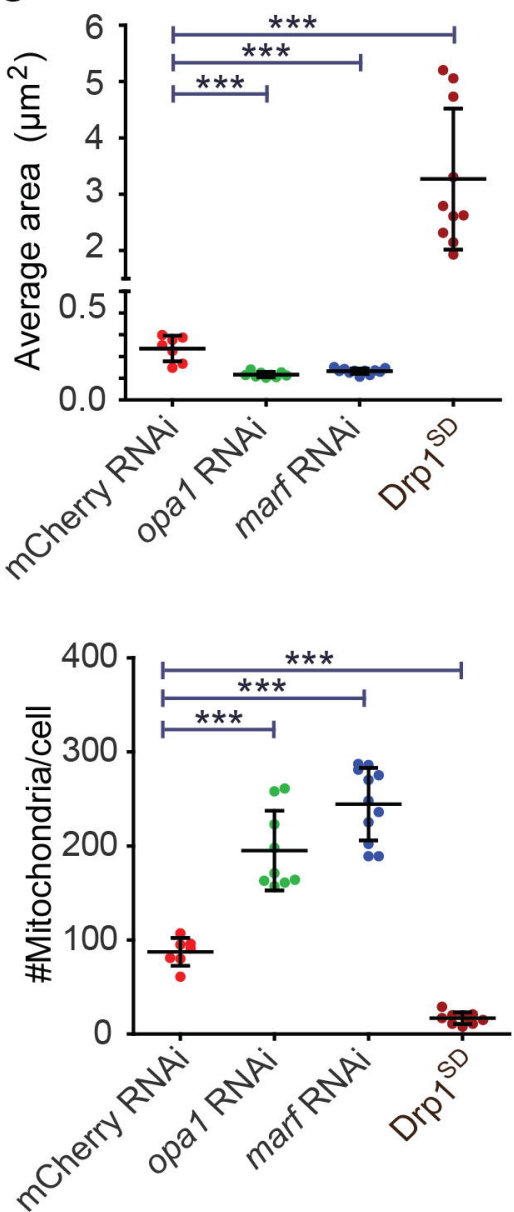


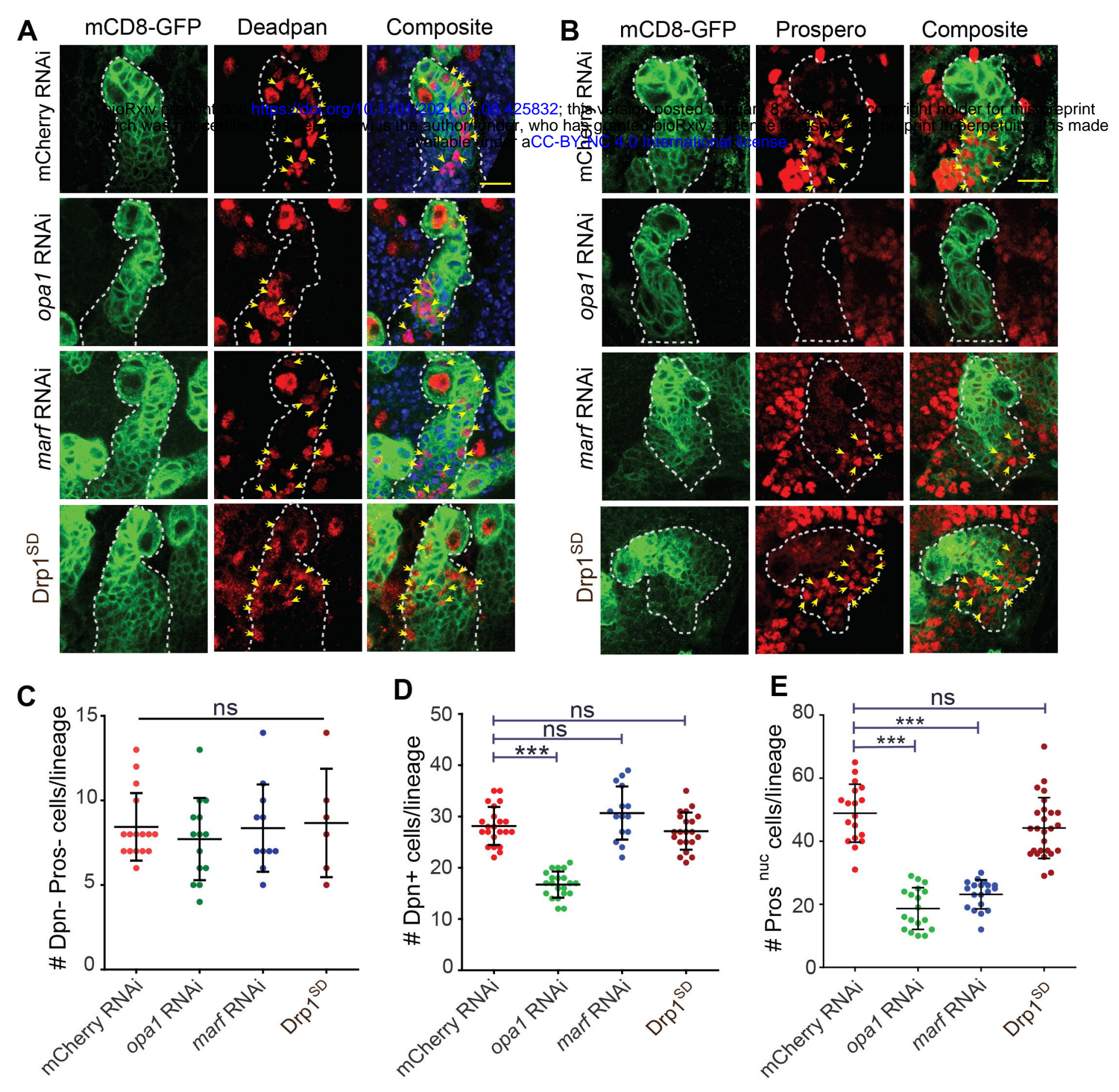




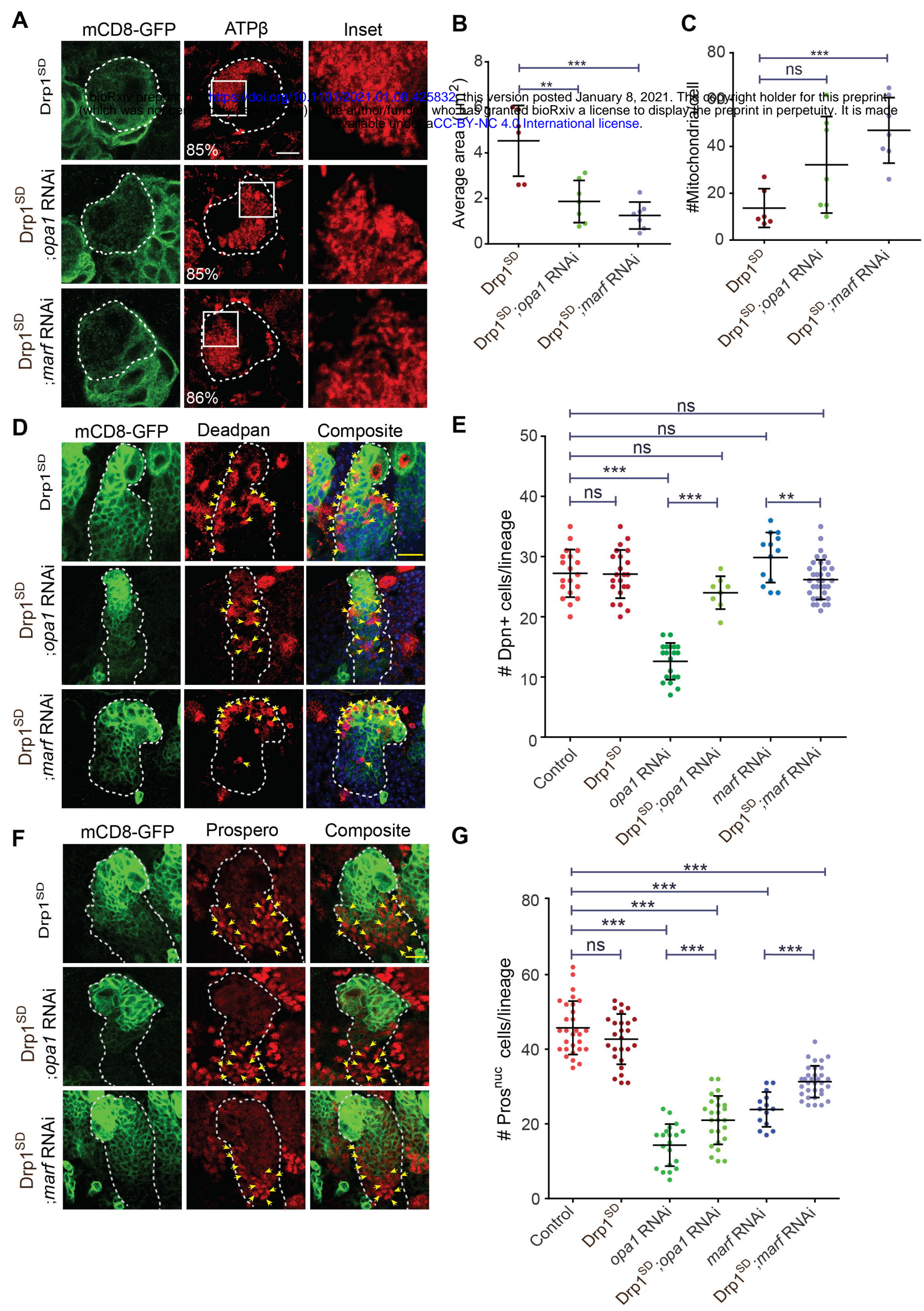




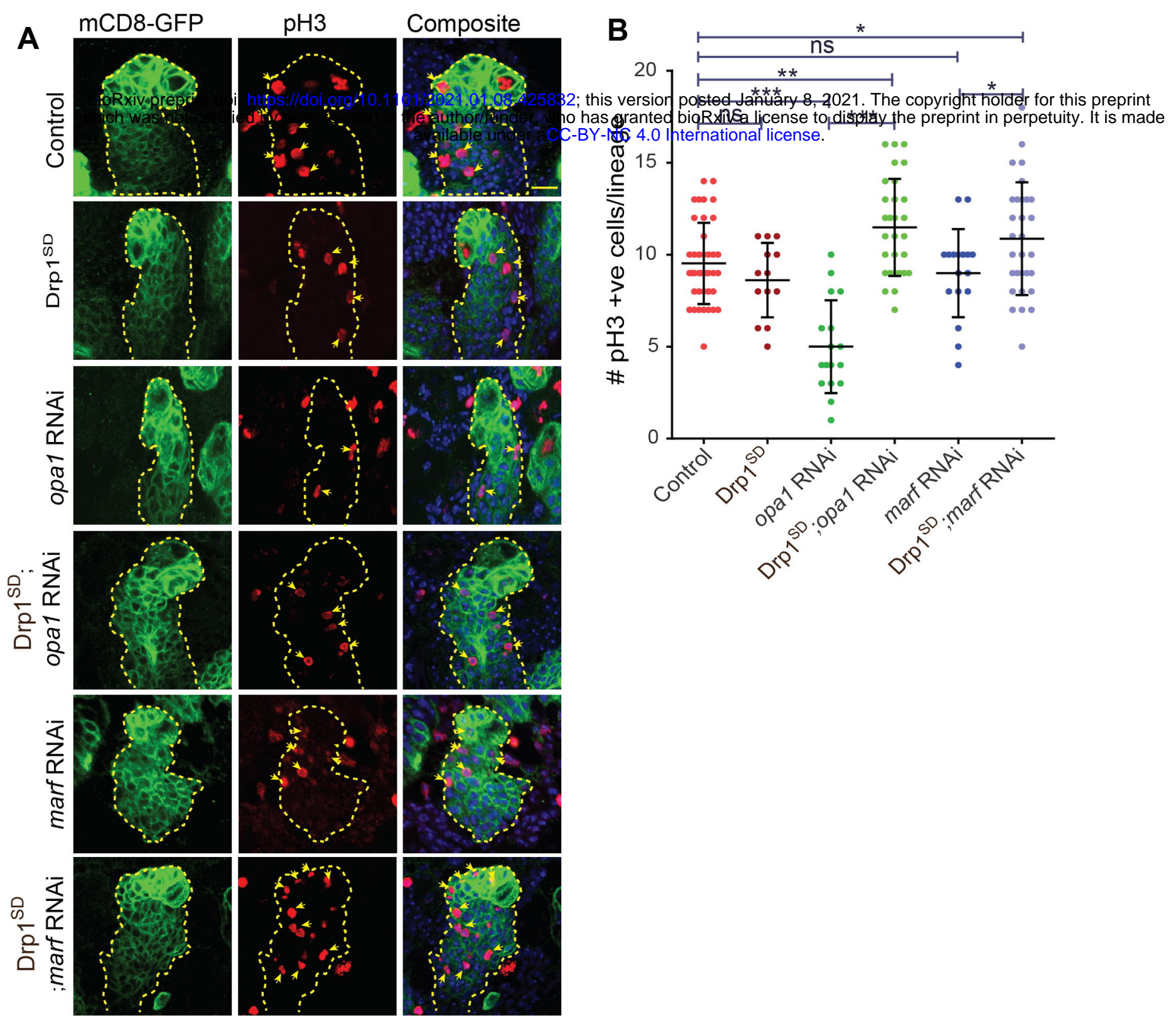



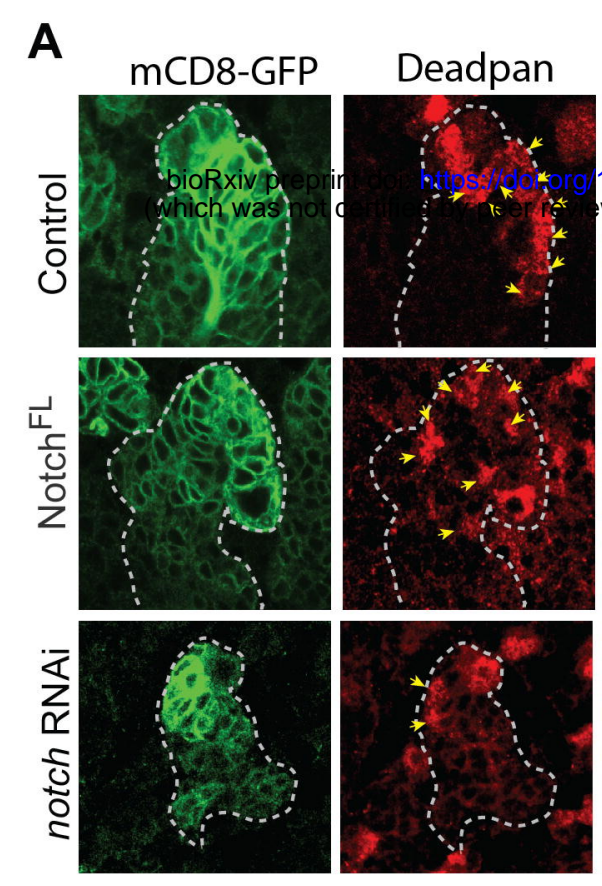

C
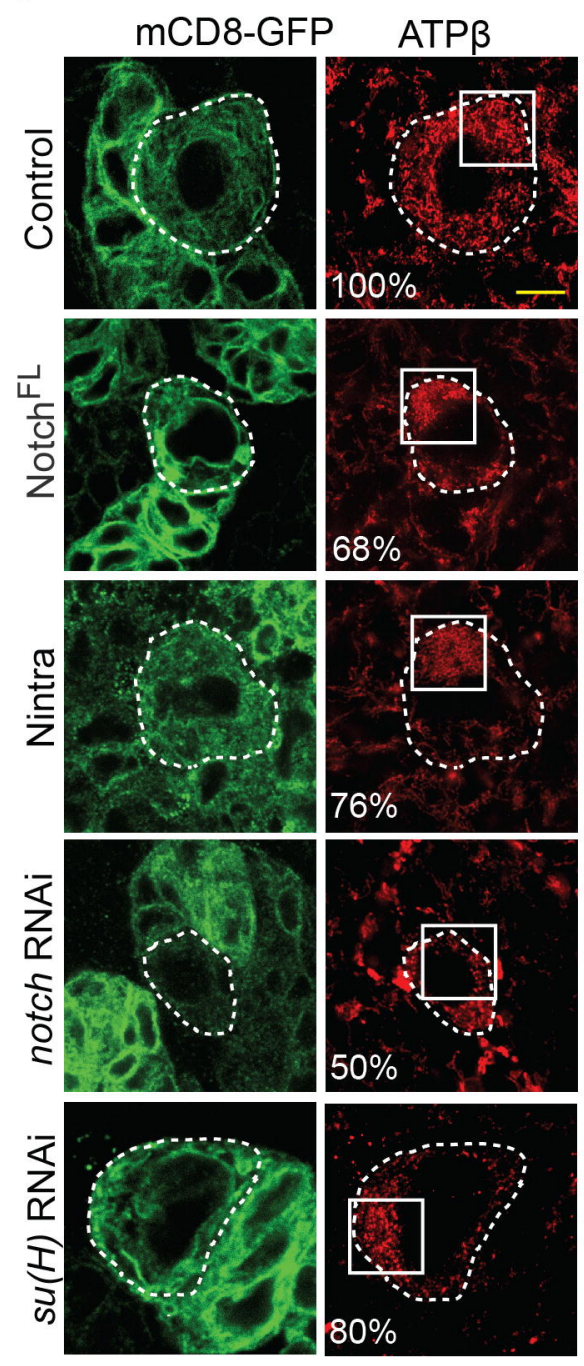

Composite
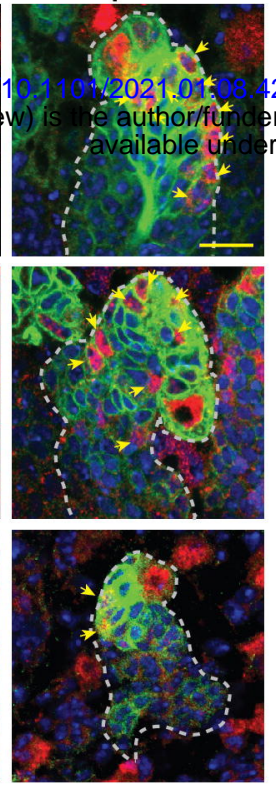

Inset
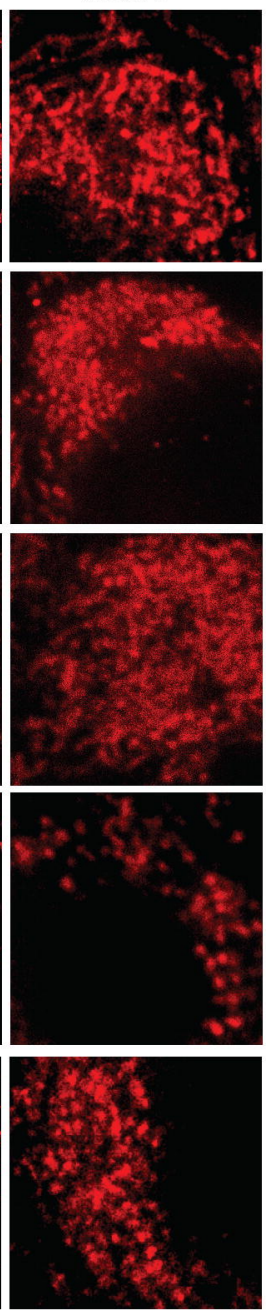

B

40 versfion posted January 8, 2021. The copyright holder for this preprint 25832; this version posted January 8,2021 . The copyright holder for this preprint

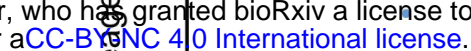

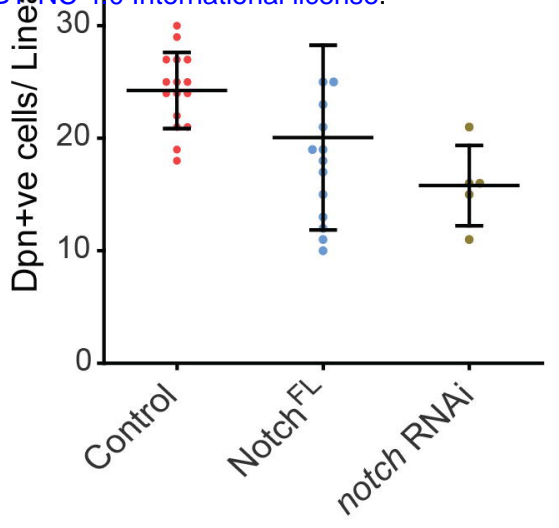

D
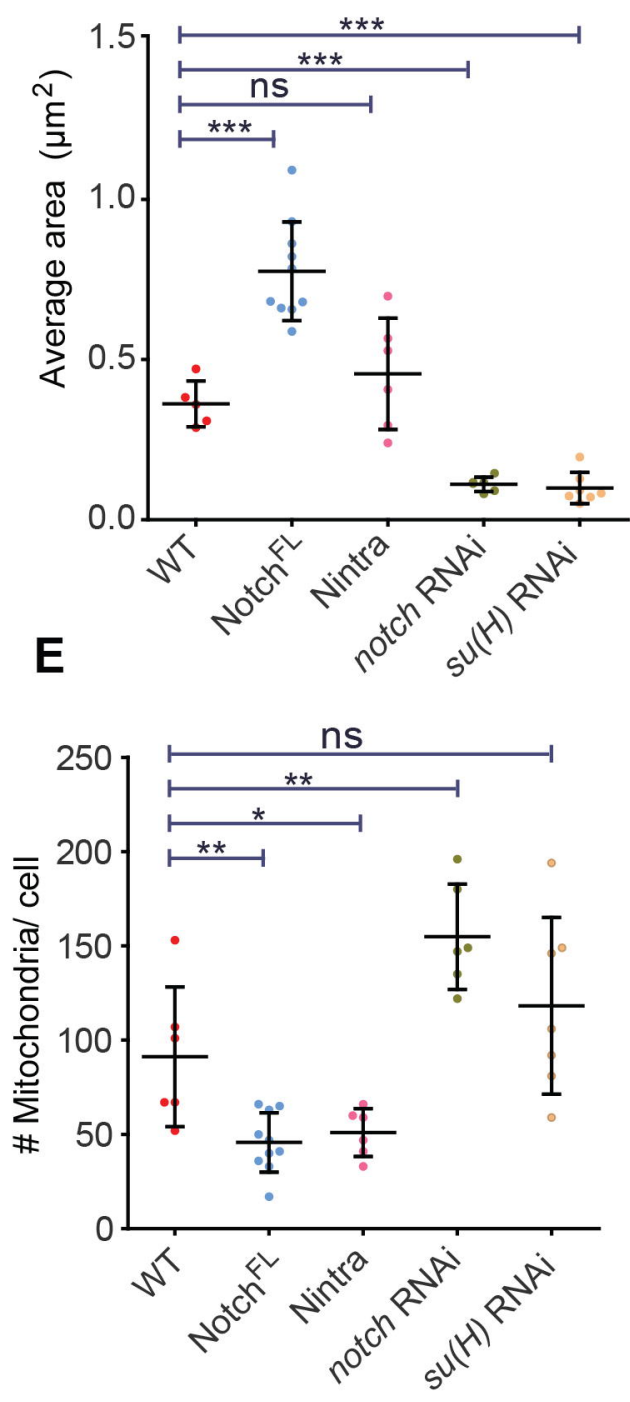
A

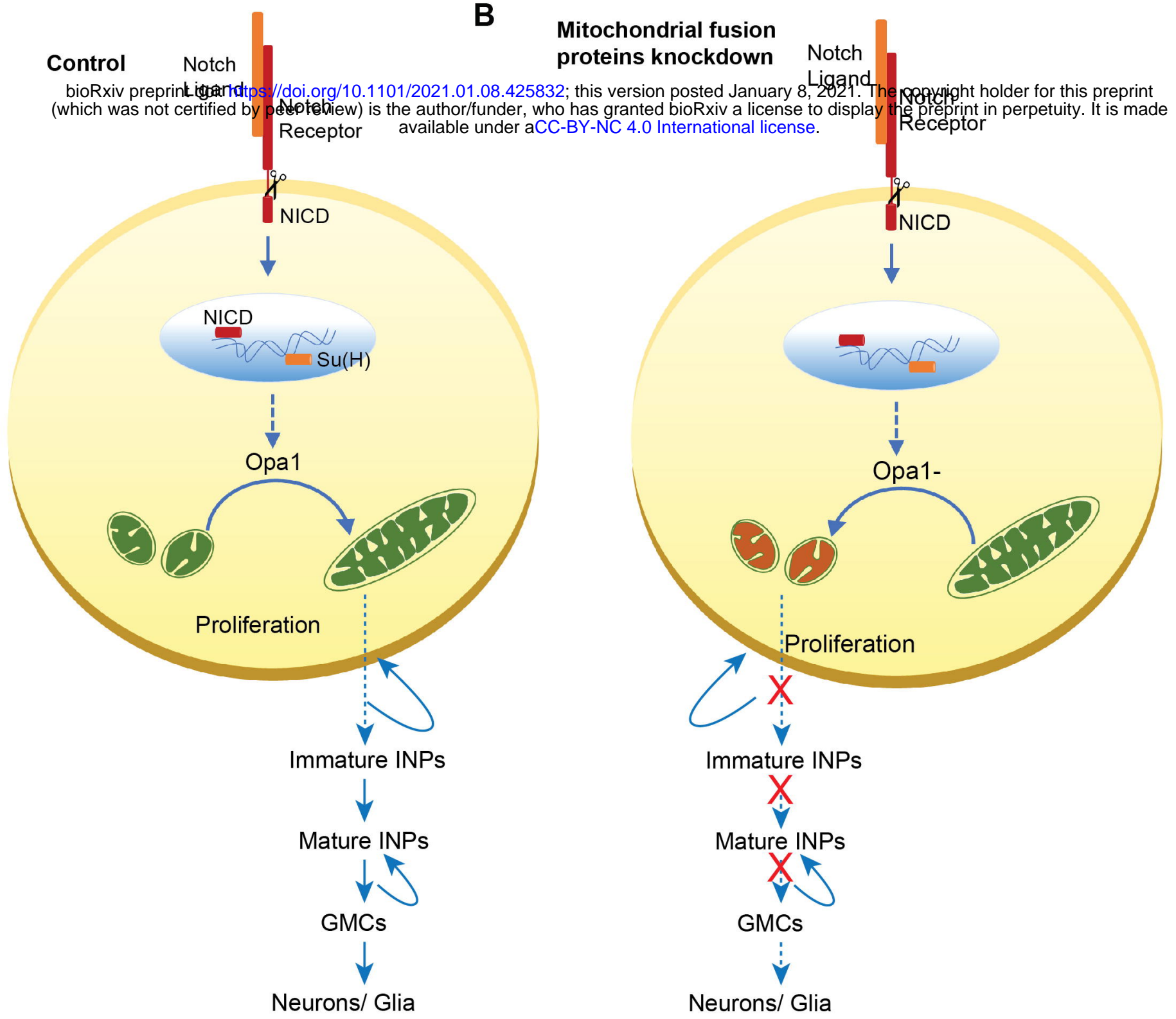

Dubal et al. 2020 Figure 8 
Worniu Gal4 Marf RNAi Ming Guo Lab* +

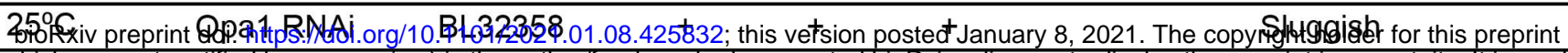

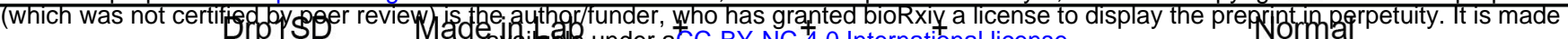

\begin{tabular}{|cccccc}
\hline \multicolumn{7}{|c}{} & & & \\
\hline Worniu Gal4 & Marf RNAi & Ming Guo Lab & + & + & + \\
\hline $29^{\circ} \mathrm{C}$ & Opa1 RNAi & BL32358 & + & + & + \\
\hline & Marf RNAi & BL67159 & + & + & + \\
\hline & Opa1 RNAi & BL67158 & + & + & + \\
\hline & Drp1SD & Made in Lab & + & + & +
\end{tabular}

\begin{tabular}{|ccccc}
\hline Pointed Gal4 & Marf RNAi & Ming Guo Lab & + & + \\
\hline $25^{\circ} \mathrm{C}$ & Opa1 RNAi & BL32358 & + & + \\
\hline & Drp1SD & Made in Lab & + & + \\
\hline Pointed Gal4 & Marf RNAi & Ming Guo Lab & + & + \\
\hline $29^{\circ} \mathrm{C}$ & Opa1 RNAi & BL32358 & + & + \\
\hline & Drp1SD & Made in Lab & + & + \\
\hline & & & & \\
\hline Inscuteable Gal4 & Marf RNAi & BL31157 & + & + \\
\hline $25^{\circ} \mathrm{C}$ & Marf RNAi & Ming Guo Lab & + & + \\
\hline & Opa1 RNAi & Ming Guo Lab & + & + \\
\hline & Opa1 RNAi & BL32358 & + & + \\
\hline & Drp1 RNAi & VDRC 44155 & + & + \\
\hline & Drp1 RNAi & BL51483 & + & + \\
\hline & & & +
\end{tabular}

$\begin{array}{cc}+ & \text { No adult emerged from pupa } \\ + & \text { No adult emerged from pupa } \\ + & \text { No adult emerged from pupa } \\ + & \text { No adult emerged from pupa } \\ + & \text { Normal }\end{array}$

\begin{tabular}{|ccccccc|}
\hline Prospero Gal4 & Marf RNAi & BL31157 & + & + & + & Normal \\
\hline $25^{0} \mathrm{C}$ & Marf RNAi & Ming Guo Lab & + & + & + & No adult emerged from pupa \\
\hline & Opa1 RNAi & Ming Guo Lab & + & + & + & Normal \\
\hline & Opa1 RNAi & BL32358 & + & + & + & No adult emerged from pupa \\
\hline & Drp1 RNAi & VDRC 44155 & + & + & + & Normal \\
\hline & Drp1 (Val20) & BL51483 & + & + & + & No adult emerged from pupa \\
\hline Elav Gal4 & Marf RNAi & Ming Guo Lab & + & + & + & Few escaper adults emerged from pupa \\
\hline $25^{\circ} \mathrm{C}$ & Opa1 RNAi & Ming Guo Lab & + & + & + & Few escaper adults emerged from pupa \\
\hline & Opa1 (Trip) & BL32358 & + & + & + & No adult emerged from pupa \\
\hline & Drp1 (Val20) & BL51483 & + & + & + & No adult emerged from pupa \\
\hline & & & & & & \\
\hline Scabrous Gal4 & Marf RNAi & BL31157 & + & + & + & Normal \\
\hline $25^{0} \mathrm{C}$ & Marf RNAi & Ming Guo Lab & + & + & + & Strong Sluggish \\
\hline & Opa1 RNAi & Ming Guo Lab & + & + & + & Normal \\
\hline & Opa1 RNAi & BL32358 & + & + & + & No adult emerged from pupa \\
\hline Drp1 RNAi & VDRC 44155 & + & + & + & Sluggish
\end{tabular}

* Deng et al., 2008

$\Delta$ Sandoval et al., 2014 
A $\quad$ B

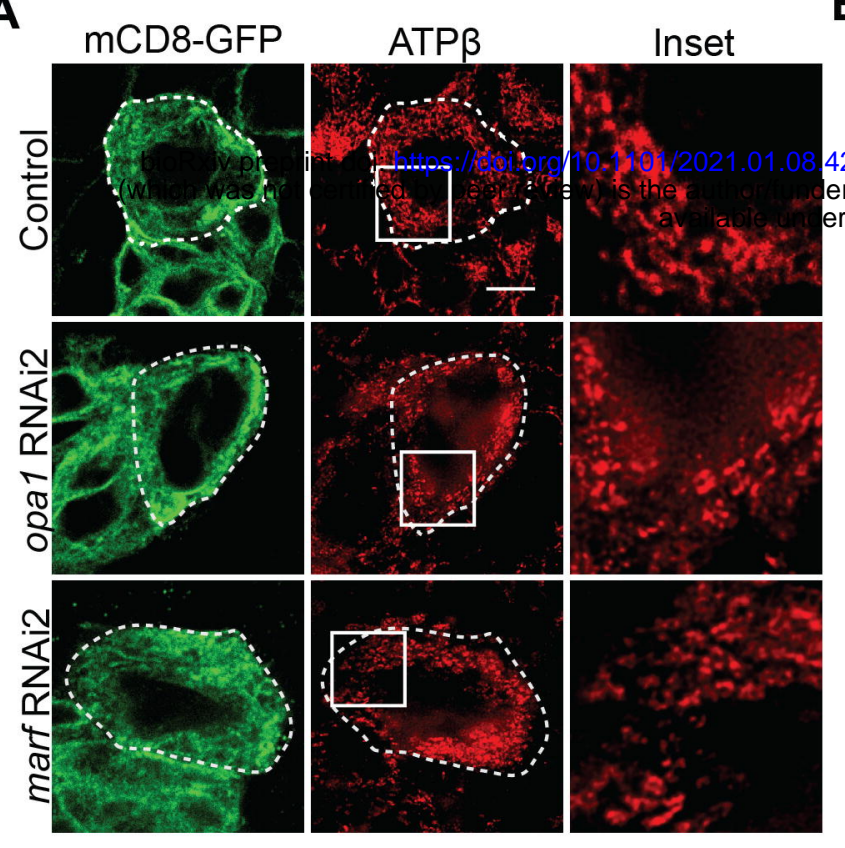

D
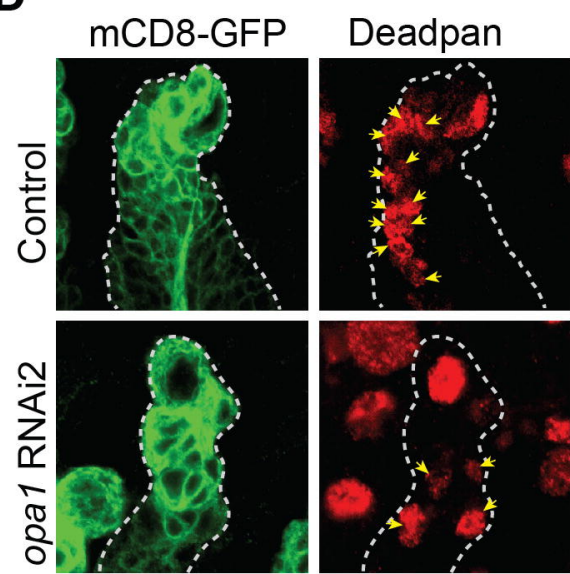

Composite
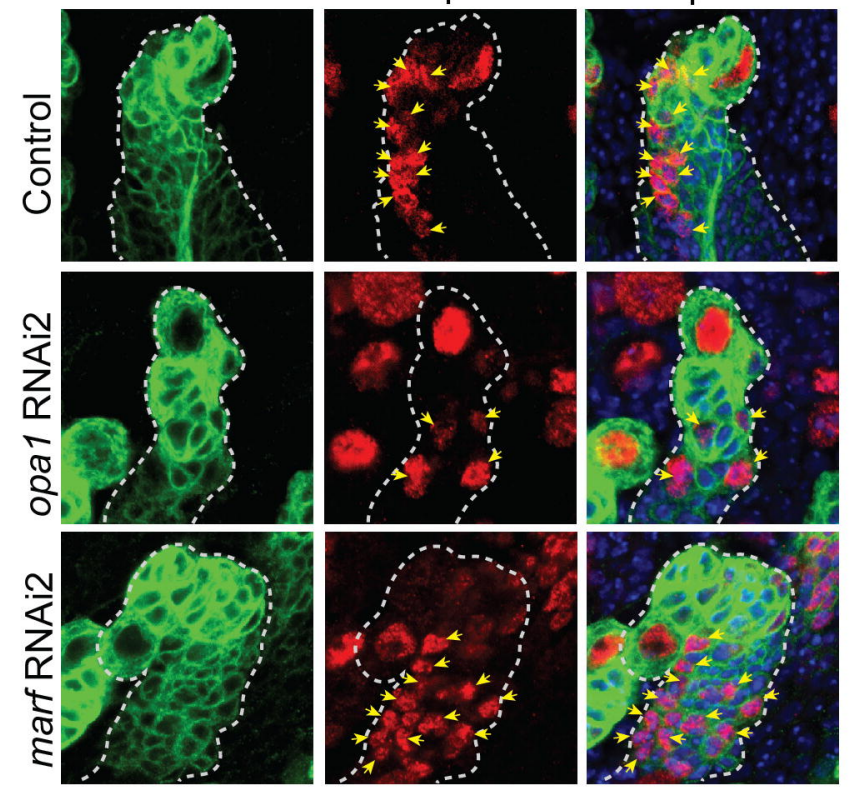

F
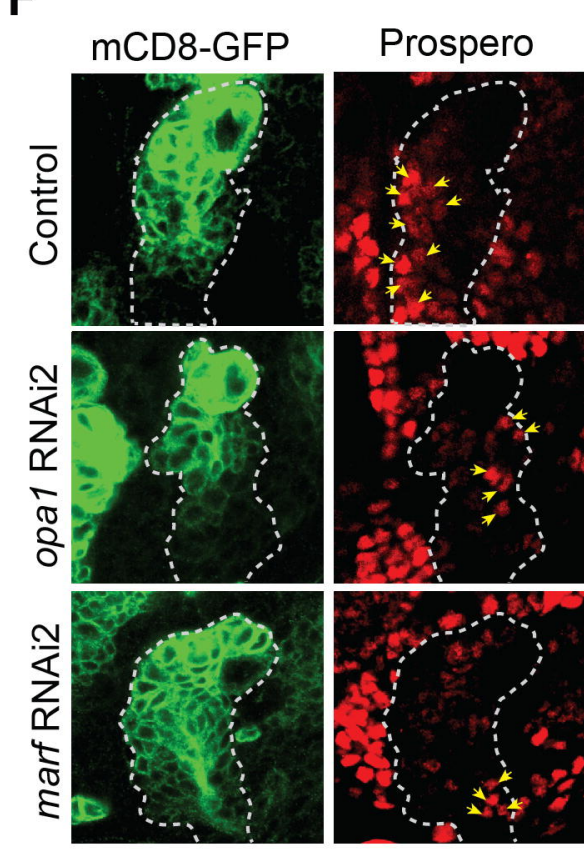

$\widetilde{\text { กิ }} 0.6 \sqrt{\frac{* * *}{1 *}}$

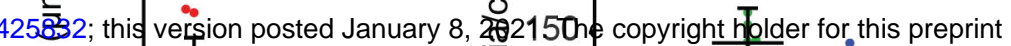
er, whochas grapted bioRxiv a license to aEC-BY-Ne 4.0 International license.

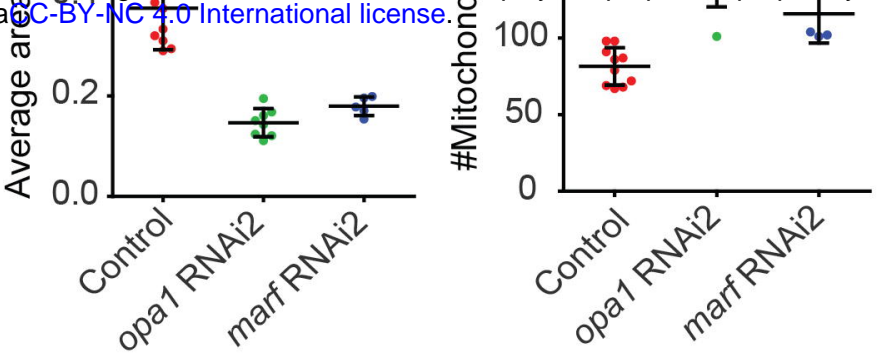

E

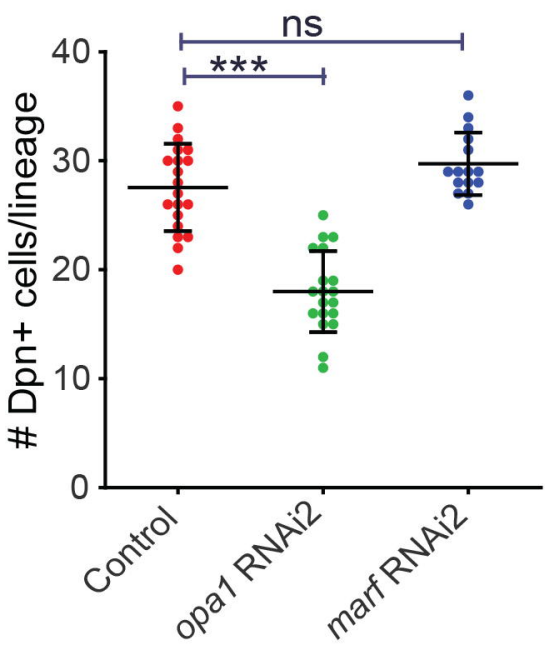

G

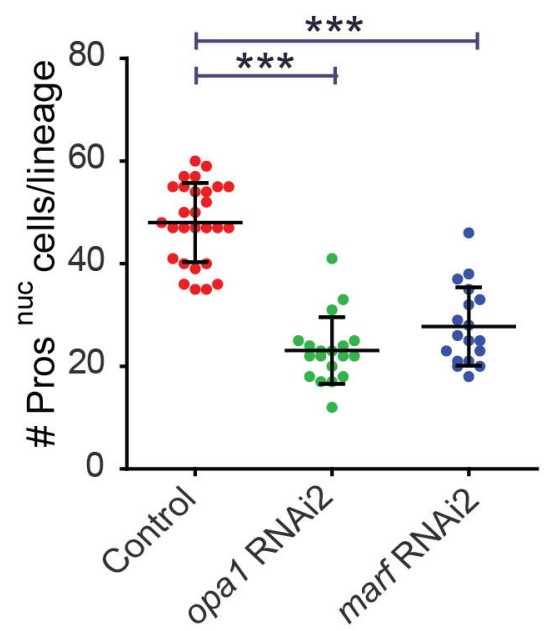




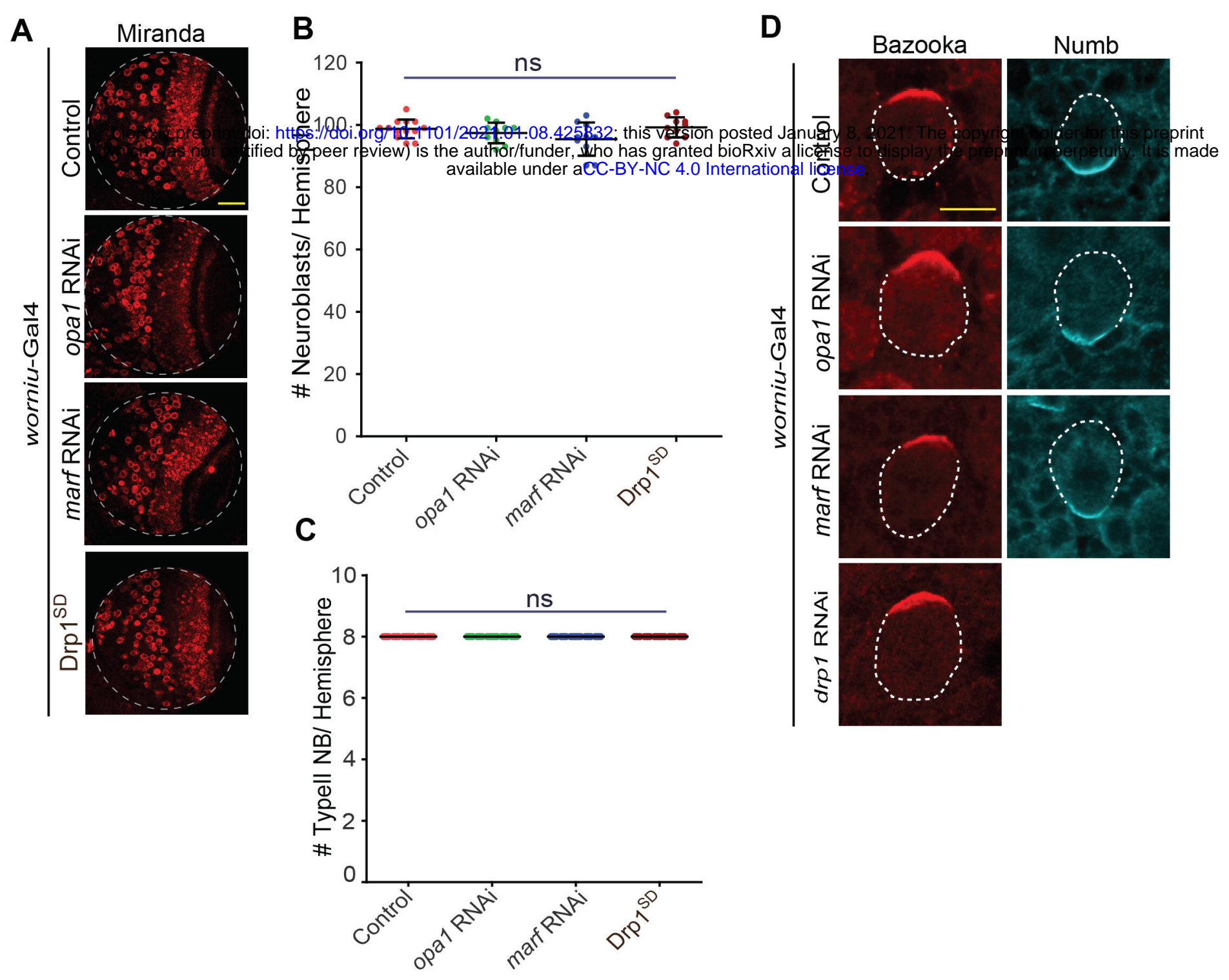

E
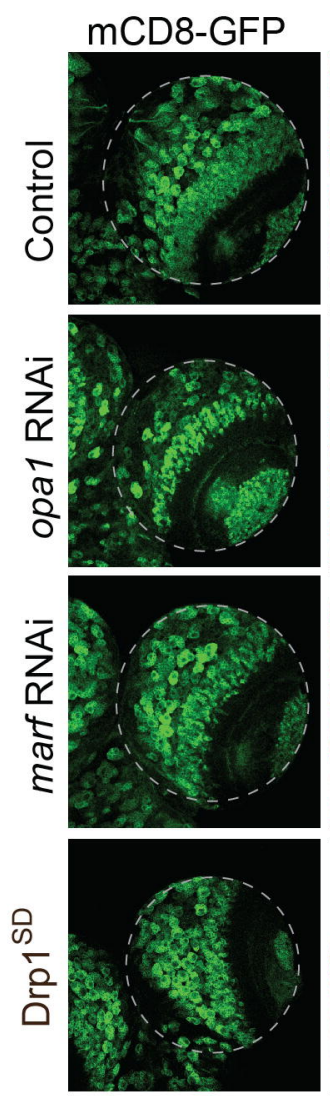
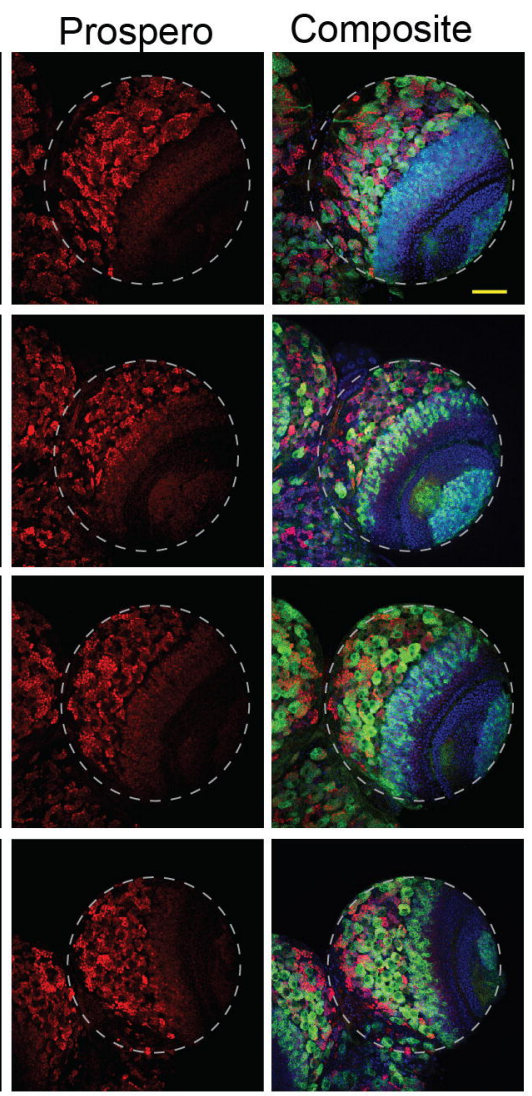

F

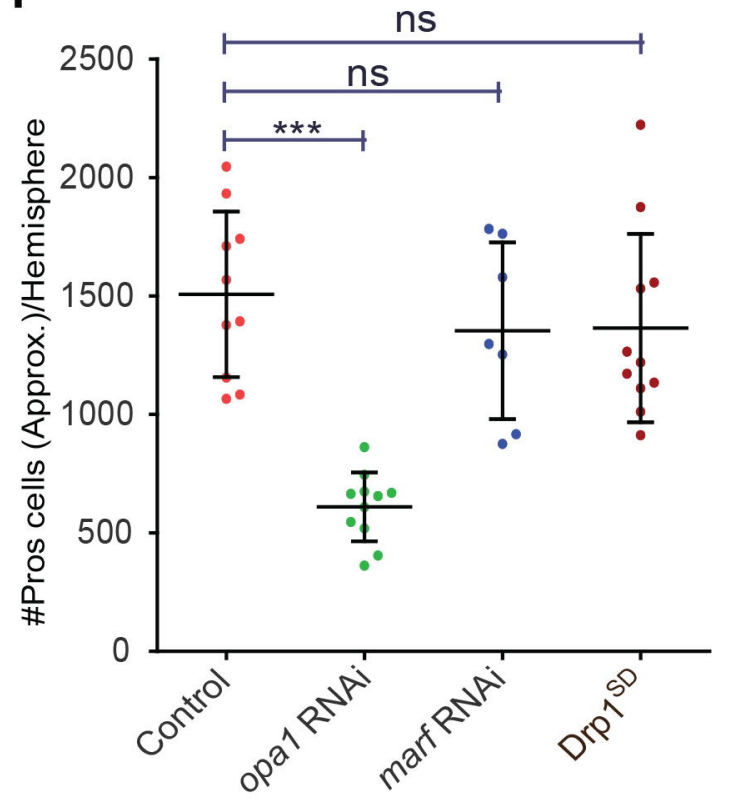




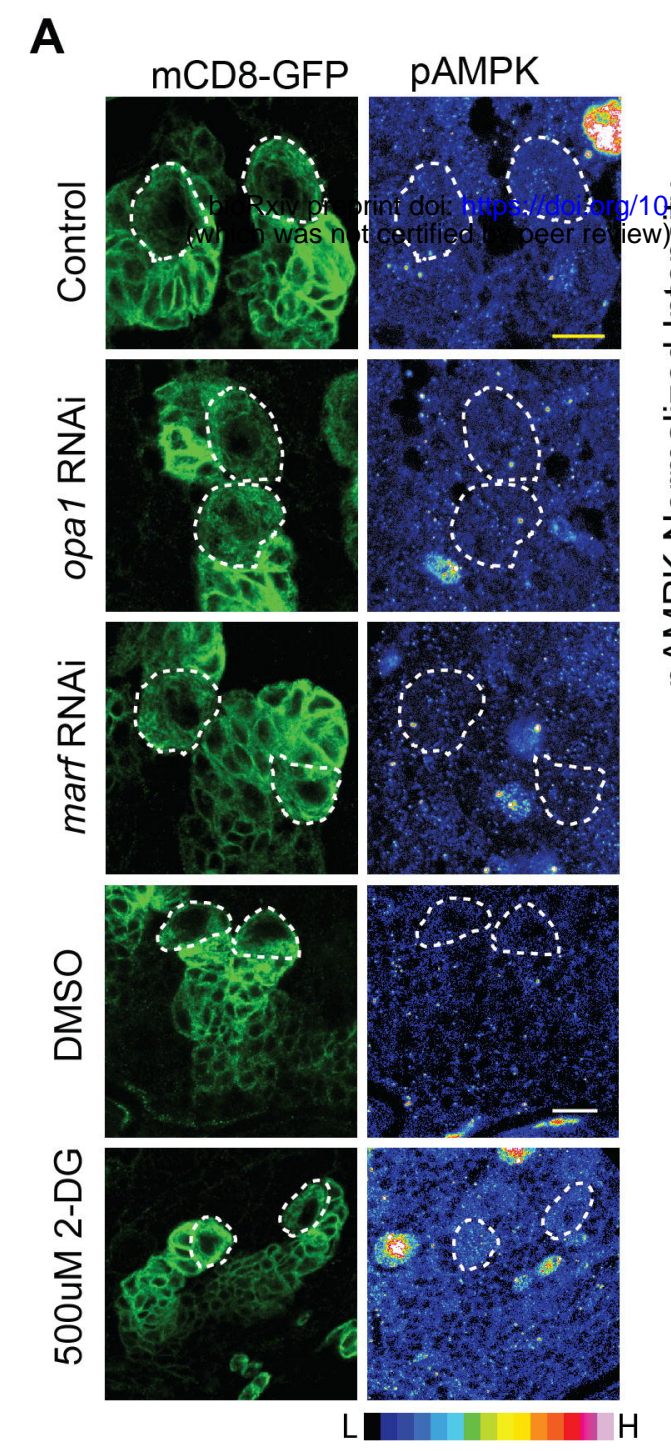

B

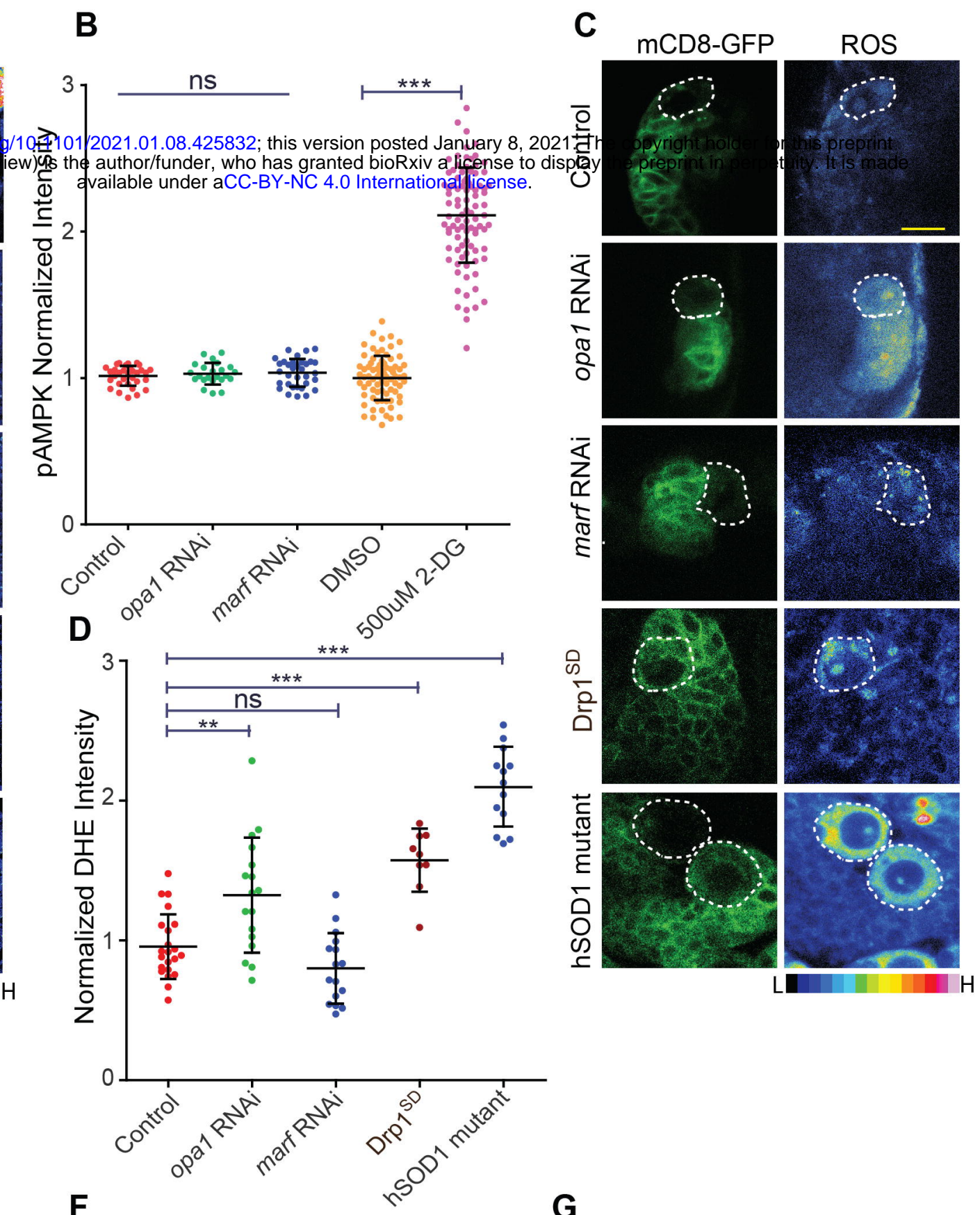

E
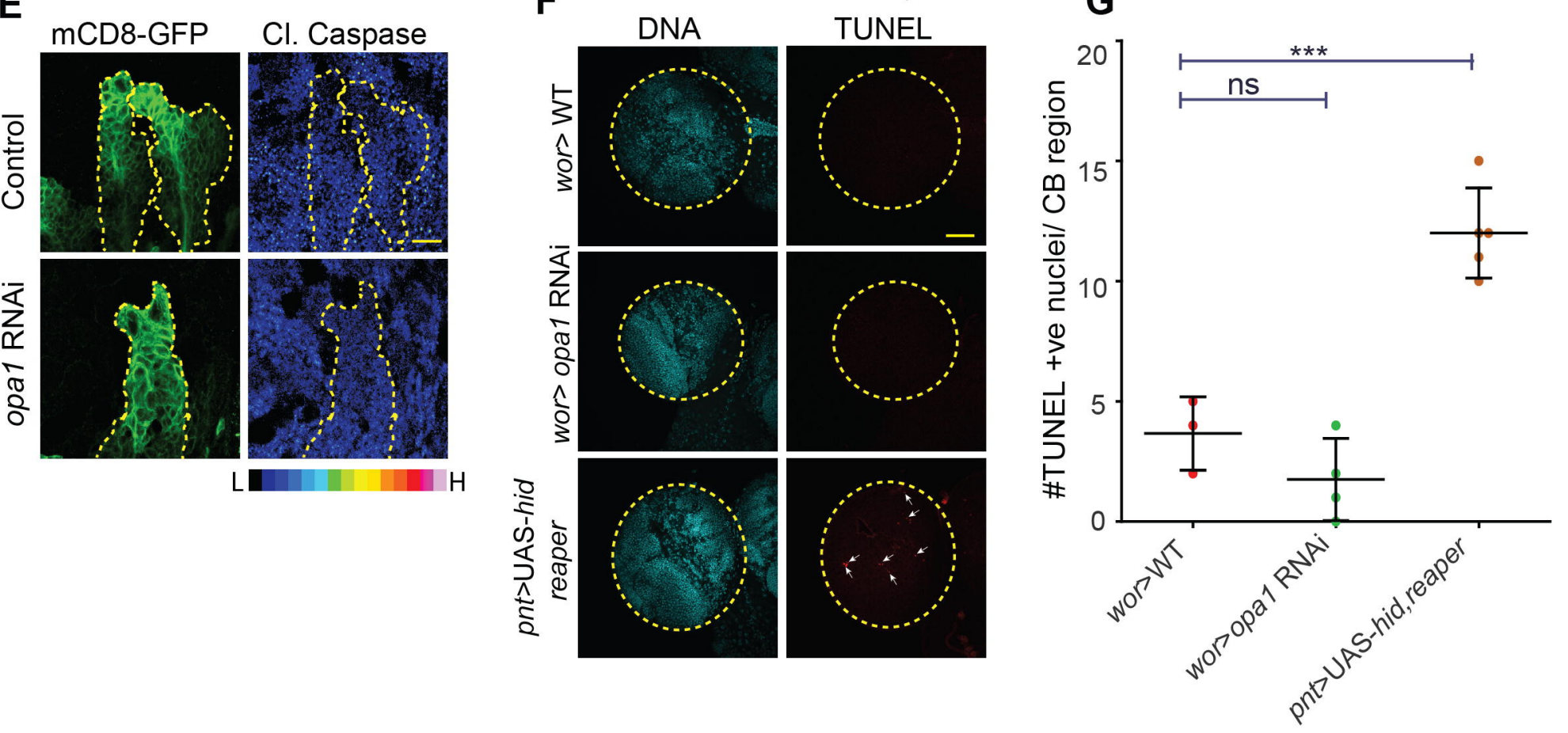
A Nintra; $\quad$ Nintra;

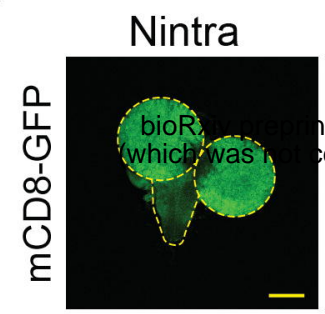
opa1 RNAi marf RNAi
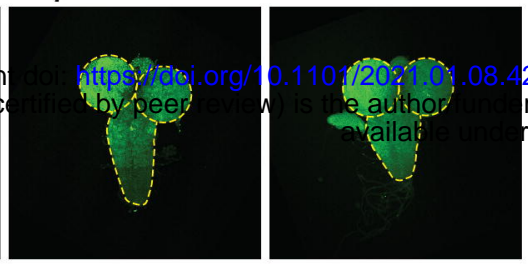

C

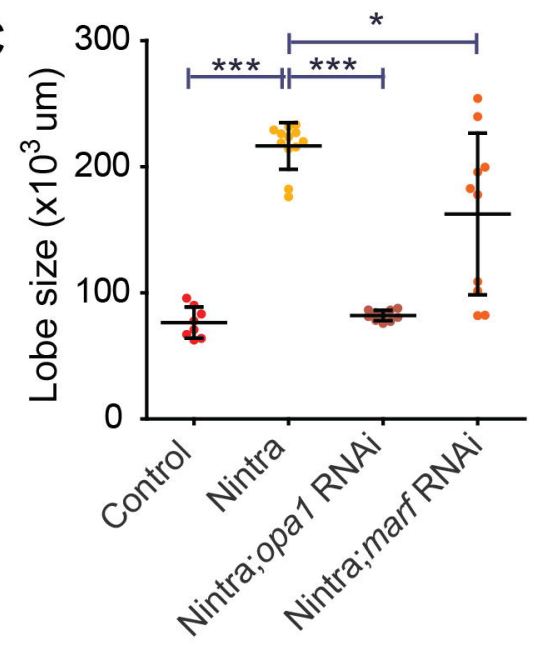

B $\underset{\pi}{\stackrel{N}{\pi}} \quad$ Nintra; Nintra;

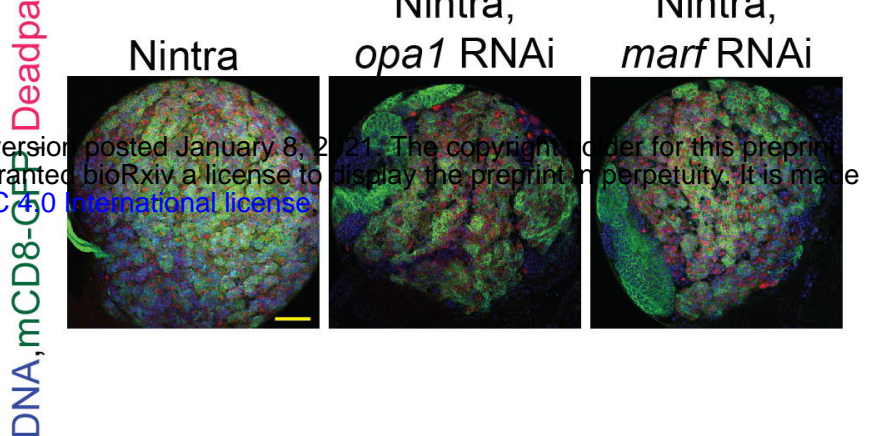

D
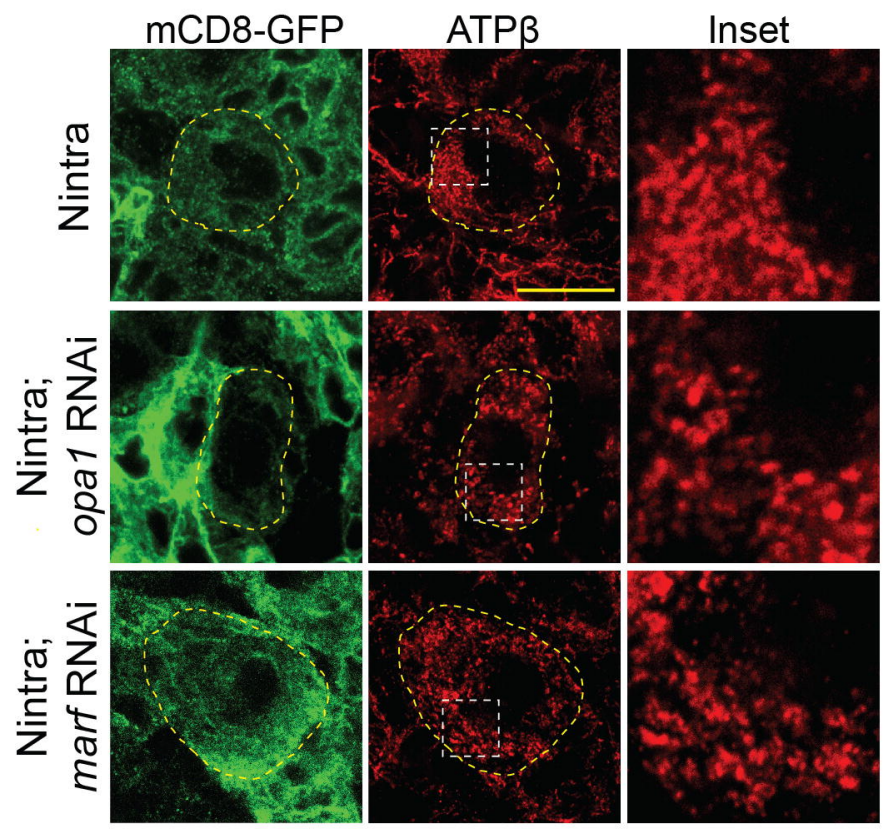